\title{
Uso de la crioterapia en la fatiga muscular post esfuerzo en individuos que realizan práctica deportiva: revisión sistemática
}

\author{
Lyda F Vallejo-Castillo', Carolina Sandoval-Cuellar², Aura C Quino- Ávila² \\ RESUMEN
}

Introducción. En la práctica deportiva, la fatiga muscular es un factor de riesgo para lesión muscular, constituyéndose en un problema que aparece cuando la carga mecánica a la cual se somete un músculo o grupo muscular es superior a la resistencia de este.

Objetivo. Describir las intervenciones con crioterapia en Fatiga Muscular post esfuerzo, en individuos que realizan práctica deportiva, a través de una Revisión Sistemática.

Método. Se realizó la búsqueda en bases de datos PUBMED/MEDLINE, EMBASE, LILACS, SCIELO, SCIENCE DIRECT, Registro Cochrane Central de Ensayos Controlados con la estrategia de búsqueda: Sports Medicine, Cryotherapy, Muscle Fatigue, Recovery of Function, Physical Exertion, Muscle Damage. La identificación y detección de las referencias fueron exportadas al gestor de referencias MENDELEY. La evaluación del Riesgo de Sesgo se realizó con el uso del software Review Manager y la calidad metodológica con lo reportado por la Base de Datos PEDro.

Resultados. Se evaluaron 19 ensayos clínicos aleatorizados en los cuales se evidencia que la crioterapia reporta cambios en la fatiga muscular desde la disminución del dolor y biomarcadores. La inmersión en agua fría fue la modalidad más efectiva para acelerar la cinética de recuperación 72 horas después del ejercicio; demostró menor dolor y mayores niveles de recuperación, percibidos a lo largo de 24 a 48 horas después del ejercicio.

Conclusión. De la totalidad de modalidades de crioterapia, la inmersión en agua fría es la que reporta mejores resultados en el proceso de recuperación de la fatiga muscular.

Palabras clave: crioterapia, fatiga muscular, recuperación de la función, medicina deportiva, esfuerzo físico, dolor muscular.

1 Indeportes Boyacá, Tunja, Colombia

2 Universidad de Boyacá, Tunja, Colombia

Correspondencia: Lyda F Vallejo-Castillo Cr 2A \# 36-85 Tunja- Boyacá - Colombia. Celular: 3144290244. Correo electrónico: lydavallejo@yahoo.es

Citar este artículo así:

Vallejo-Castillo LF, Sandoval-Cuellar C, Quino- Ávila AC. Uso de la crioterapia en la fatiga muscular post esfuerzo en individuos que realizan práctica deportiva: Revisión Sistemática.Revista Investig Salud Univ Boyacá. 2019;6(2): 71-98. doi: https://doi.org/10.24267/23897325.416 


\title{
Use of cryotherapy in post-effort muscle fatigue in individuals who practice sports: systematic review
}

\begin{abstract}
Introduction. In sports practice, muscle fatigue is a risk factor for muscle injury becoming a problem that appears when the mechanical load to which a muscle or muscle group is subjected is greater than its resistance.

Objective. Describe post-effort Muscular Fatigue in active Men and Women who perform sports practice against interventions with cryotherapy, through a Systematic Review.

Method. PUBMED / MEDLINE, EMBASE, LILACS, SCIELO, SCIENCE DIRECT, Central Cochrane Registry of Controlled Trials with the search strategy were searched: Sports Medicine, Cryotherapy, Muscle Fatigue, Recovery of Function, Physical Exertion, Muscle Damage The identification and detection of references were exported to the MENDELEY reference manager. The Bias Risk assessment was performed with the use of Review Manager software and methodological quality as reported by the PEDro Database.
\end{abstract}

Results. 19 randomized clinical trials were evaluated in which it is evident that cryotherapy reports changes in muscle fatigue since the decrease in pain, and biomarkers. Cold water immersion was the most effective modality to accelerate recovery kinetics 72 hours after exercise; showed less pain and higher levels of recovery perceived over 24 to 48 hours after exercise.

Conclusion. Of all the cryotherapy modalities, the immersion in cold water is the one that reports the best results in the process of recovery of muscular fatigue.

Key words. Cryotherapy, Muscle fatigue, Recovery of Function, Sports Medicine, physical exertion, muscle pain. 


\section{Uso da crioterapia na fadiga muscular pós-esforço em indivíduos que realizam prática esportiva: revisão sistemática}

\section{RESUMO}

Introdução. Na prática esportiva, a fadiga muscular é um fator de risco para a lesão muscular, tornando-se um problema que ocorre quando a carga mecânica à qual um músculo ou grupo muscular é submetido é superior à resistência do músculo.

Objetivo. Descrever as intervenções com crioterapia na fadiga muscular pós-esforço, em indivíduos que realizam prática esportiva, por meio de uma revisão sistemática.

Método. Foi realizada uma busca nas bases de dados PUBMED/MEDLINE, EMBASE, LILACS, SCIELO, SCIENCE DIRECT, Registro Central Cochrane de Ensaios Controlados com a estratégia de busca: Medicina Esportiva, Crioterapia, Fadiga Muscular, Recuperação da Função, Esforço Físico, Dano Muscular. A identificação e deteç̧ão das referências foram exportadas para o gerenciador de referência do MENDELEY. A avaliação do risco de viés foi realizada com o uso do software Review Manager de revisão e qualidade metodológica com a relatada pelo banco de dados PEDro.

Resultados. Dezenove ensaios clínicos randomizados foram avaliados nos quais se evidencia que a crioterapia relata alterações na fadiga muscular desde a diminuição da dor e dos biomarcadores. A imersão em água fria foi a modalidade mais eficaz para acelerar a cinética de recuperação 72 horas após o exercício; demonstrou menos dor e níveis mais altos de recuperação, percebidos entre 24 e 48 horas após o exercício.

Conclusão. De todas as modalidades de crioterapia, a imersão em água fria é a que proporciona os melhores resultados no processo de recuperação da fadiga muscular.

PALAVRAS-CHAVE: crioterapia, fadiga muscular, recuperação de desempenho, medicina esportiva, esforço físico, dor muscular. 


\section{INTRODUCCIÓN}

La práctica de actividades físico-deportivas y el deporte competitivo o profesional utilizan gran parte de su tiempo en la recuperación muscular; en este sentido, los actuales modelos de competición y el creciente auge de la práctica deportiva han considerado de gran importancia los aspectos relacionados con la recuperación post esfuerzo (RECP) y la disminución de la fatiga muscular (FAT) (1), entendiendo el efecto del entrenamiento como una mejora en el rendimiento físico que se produce como resultado de la fatiga producida por las de cargas de trabajo unida a los procesos de recuperación del organismo (2). La RECP y disminución de la FAT están influenciadas por varios métodos, y modalidades físicas siendo la crioterapia uno de ellos. Durante décadas, diferentes formas de crioterapia, como las inmersiones totales o parciales del cuerpo en agua fría (CWI), aplicación local o masaje con hielo y compresas frías, se han utilizado en diferentes modalidades deportivas para hacer frente a la RECP, a la FAT y al dolor muscular de aparición tardía (DOMS) (3).

Atendiendo a la temática, se reportan revisiones sistemáticas y meta análisis como el de Dupuy (4), en el cual evaluaron el impacto de las técnicas de recuperación en el DOMS, fatiga, daño muscular y marcadores inflamatorios después del ejercicio físico, reportando que el masaje es el método más efectivo para la recuperación del
DOMS y la fatiga, en términos de daño muscular y marcadores inflamatorios, las técnicas más efectivas fueron el masaje y la exposición al frío. Así mismo, Hohenauer (5) determinó los posibles efectos de diferentes aplicaciones de enfriamiento, en comparación con las estrategias pasivas post ejercicio sin enfriamiento, sobre las características de recuperación después de varios protocolos de ejercicio; revelando que el enfriamiento y especialmente las inmersiones en agua fría afectaron significativamente los síntomas de DOMS, en comparación con las condiciones de control. Por último, Torres (6) evalúo si algunas modalidades utilizadas actualmente en fisioterapia como el masaje, la crioterapia, el estiramiento y el ejercicio de baja intensidad son efectivas para tratar los signos y síntomas de daño muscular inducido por el ejercicio; de esta forma, evidenció que el masaje era la única intervención con efectos positivos, el cual reducía el dolor a las $24 \mathrm{~h}$ y mejoraba la recuperación muscular; de la misma manera, los estudios citados mencionan la falta de evidencia para apoyar el uso de crioterapia, estiramientos y ejercicio de baja intensidad.

Respecto a la fatiga muscular, esta se define como la imposibilidad de generar una fuerza requerida o esperada, producida o no por un ejercicio precedente, la cual se describe como un estado funcional que desencadena una respuesta homeostática con el objetivo de proteger al organismo; además, es transitoria y reversible, por lo que 
produce una necesidad ineludible de disminuir la intensidad de un esfuerzo o, incluso, de concluirlo o terminarlo (7).

La crioterapia es la aplicación local o sistémica de frío con fines terapéuticos y es una de las modalidades físicas más usadas en fisioterapia; históricamente se ha utilizado, de manera prioritaria, en el tratamiento de lesiones agudas. La disminución de la temperatura es el efecto principal a partir del cual se generan cambios en diferentes tejidos $y / 0$ sistemas, esta depende del tipo, tiempo de aplicación y capacidad para absorber y transferir calor a los tejidos (8).

La crioterapia es uno de los medios más utilizados para el tratamiento del dolor producto de disfunciones de origen musculoesquelético. La evidencia, tanto neurofisiológica como clínica, sugiere que su uso reduce el flujo sanguíneo local, la tasa metabólica de los tejidos y la velocidad de conducción nerviosa; estos efectos tisulares son los responsables de los resultados terapéuticos atribuidos a la crioterapia, entre los cuales están: disminución del dolor, de la reacción inflamatoria y retraso en la formación de edema. La reducción del diámetro del lumen del vaso sanguíneo causa una disminución significativa del flujo de sangre del tejido subcutáneo, con lo cual se aminora la filtración de fluido al intersticio y al edema secundario, se controla la inflamación y se disminuyen tanto el dolor como la liberación de sustancias vasoactivas (9).
El efecto analgésico de la crioterapia se fundamenta en algunos cambios evidenciables en la electrofisiología neuromuscular, además, se asocia a la disminución local de la temperatura cutánea, produciendo un retraso tanto en la apertura como en el cierre de los canales de sodio; de este modo, hay un enlentecimiento de las corrientes de sodio responsables de la despolarización de las fibras nerviosas y/o musculares, lo que se traduce finalmente en una reducción de la velocidad de conducción nerviosa del axón. El hecho de tener un periodo refractario más largo condiciona, además, una disminución del potencial de acción que, sumada a la disminución de la frecuencia de descarga del nociceptor, explicaría el aumento del umbral del dolor y el potencial efecto hipoalgésico atribuido a la crioterapia. La disminución de la tasa metabólica también reduce la demanda de oxígeno de los tejidos y reduce al mínimo las posibilidades de una herida hipóxica secundaria de los tejidos debido a la isquemia. Adicionalmente, se ha postulado que la reducción del flujo sanguíneo y la disminución del edema disminuiría la compresión mecánica de estructuras vasculonerviosas sensibles a la presión y, de esta forma, se produciría un alivio del dolor por causa mecánica (9).

Sustentados en la importancia de esta modalidad física el presente trabajo estableció como propósito identificar ¿Cuáles son las intervenciones con crioterapia en Fatiga Muscular post esfuerzo, en individuos que realizan práctica deportiva? 


\section{MATERIALES Y MÉTODOS}

Se establecieron los criterios según la estrategia PICO como aparece en la tabla 1, y se determinaron como criterios de inclusión y exclusión lo expresado en la tabla 2.

Tabla 1. Elementos de la pregunta PICO

\begin{tabular}{ll}
\hline Población & $\begin{array}{l}\text { Hombres y mujeres activos que realizan práctica } \\
\text { deportiva con diferentes niveles de entrena- } \\
\text { miento, con edades entre } 15 \text { y } 35 \text { años. }\end{array}$ \\
\hline Intervención & $\begin{array}{l}\text { Crioterapia (inmersión en agua fría, crioterapia } \\
\text { local con bolsas de hielo, compresas térmicas y } \\
\text { crio cámara basada en nitrógeno gaseoso). }\end{array}$ \\
\hline Resultados & $\begin{array}{l}\text { Secundarios: FATIGA MUSCULAR, especí- } \\
\text { ficamente referida por los resultados obtenidos } \\
\text { lación de colocación de ultrasonido - placebo, } \\
\text { reposo o descanso, drenaje linfático manual, } \\
\text { fotobiomodulación, estiramientos estáticos } \\
\text { y balísticos, recuperación activa y ecografía } \\
\text { simulada. }\end{array}$ \\
\hline & $\begin{array}{l}\text { isométrica, B. Inflamación, C. Masa muscular } \\
\text { según segmento corporal, D. Salto vertical, E. } \\
\text { Rango de Movimiento. }\end{array}$ \\
\hline & Primarios: FATIGA MUSCULAR, específica- \\
mente referida por los resultados obtenidos de \\
la evaluación del dolor muscular y los marcado- \\
res bioquímicos.
\end{tabular}

Fuente: Las autoras
Tabla 2. Criterios de inclusión y exclusión

\begin{tabular}{cl}
\hline Criterios & $\begin{array}{l}\text { Ensayos clínicos controlados aleatorios } \\
\text { con diseños paralelos de dos, tres y has- } \\
\text { ta cinco grupos, y diseños cruzados. }\end{array}$ \\
\hline de inclusión & $\begin{array}{l}\text { Estudios realizados con animales, estu- } \\
\text { dios que no tengan firma de consenti- } \\
\text { miento informado ni consideraciones } \\
\text { bioéticas. }\end{array}$ \\
\hline
\end{tabular}

\section{Búsqueda de Artículos}

La búsqueda que fue realizada entre enero de 2019 y junio de 2019, en las bases de datos de PUBMED/MEDLINE, EMBASE, LILACS, SCIELO, SCIENCE DIRECT, Registro Cochrane Central de Ensayos Controlados (CENTRAL) por un revisor cegado. Se tuvieron en cuenta las recomendaciones de Robinson y Dickersin (10) para una estrategia de búsqueda altamente sensible en la recopilación de ensayos clínicos en Pubmed. En la estrategia de búsqueda se combinaron los siguientes términos: ["Sports Medicine"[Mesh]) AND "Cryotherapy"[Mesh]) AND "Muscle Fatigue"[Mesh]], ["Cryotherapy"[Mesh]) AND "Recovery of Function"[Mesh]) AND "Muscle Fatigue"[Mesh]) AND "Sports Medicine"[Mesh]], [(("Cryotherapy"[Mesh]) AND "Recovery of Function"[Mesh]) AND "Muscle Fatigue"[Mesh]] y [ (("Cryotherapy"[Mesh]) AND "Physical Exertion"[Mesh]) AND "Muscle Fatigue"[Mesh] muscle damage]. Se presentaron limitaciones idiomáticas, atendiendo a que solo se incluyeron artículos en idioma inglés y portugués. Las 
autoras determinan adicionalmente la inclusión de ECA que expresarán el cumplimiento de las consideraciones éticas.

\section{Identificación de los Estudios y Extracción de Datos}

Un revisor cegado realizó las búsquedas electrónicas (identificación y detección); las referencias identificadas fueron exportadas al gestor de referencias MENDELEY, el cual realizó automáticamente la eliminación de duplicados con el uso de metadatos como: título, autor y año; posteriormente, dos autores y un nuevo integrante identificado realizaron la revisión de los artículos a texto completo, diligenciando matriz de extracción en la cual se establecieron variables relacionadas con el estudio y cumplimiento de criterios de inclusión del mismo. En cuanto a las discrepancias que se generaron, estas se resolvieron por consenso. Una vez seleccionados los estudios, el autor extrajo las características específicas de cada estudio (Tabla 3). El proceso de selección de los estudios se evidencia a través del diagrama de flujo, de acuerdo con la declaración de PRISMA (11) (Figura 1).

\section{Evaluación del Riesgo de Sesgo y Calidad Metodológica}

La evaluación de riesgo de sesgo se estableció según en los lineamientos del Manual Cochrane de revisiones sistemáticas, el cual evalúa el Sesgo de selección, de realización, de detección, de desgaste, de notificación y otros sesgos; por otra parte, esta evaluación utilizó el software Review Manager 5.3 y la calidad metodológica se evaluó mediante el Physiotherapy Evidence Database, PEDro, el cual establece diez criterios de medición (12), teniendo en cuenta los siguientes: generación de secuencia, el ocultamiento de la asignación, el cegamiento de resultados primarios, el adecuado abordaje a los datos de resultados incompletos, el riesgo de informe selectivo, y otros riesgos de sesgo.

\section{RESULTADOS}

Esta revisión sistemática fue reportada acorde a los lineamientos descritos en la declaración PRISMA (13) y en el Manual Cochrane para la conducción de Revisiones Sistemáticas de Intervenciones versión 5.1 (14).

\section{Extracción de Datos}

Una vez se obtuvieron los estudios a incluir, el autor, de forma independiente, extrajo las características específicas de cada estudio. El proceso de selección de los estudios se observa a través del diagrama de flujo de acuerdo con lo establecido en la declaración de PRISMA (Figura 1). 
Figura 1. Diagrama de flujo PRISMA

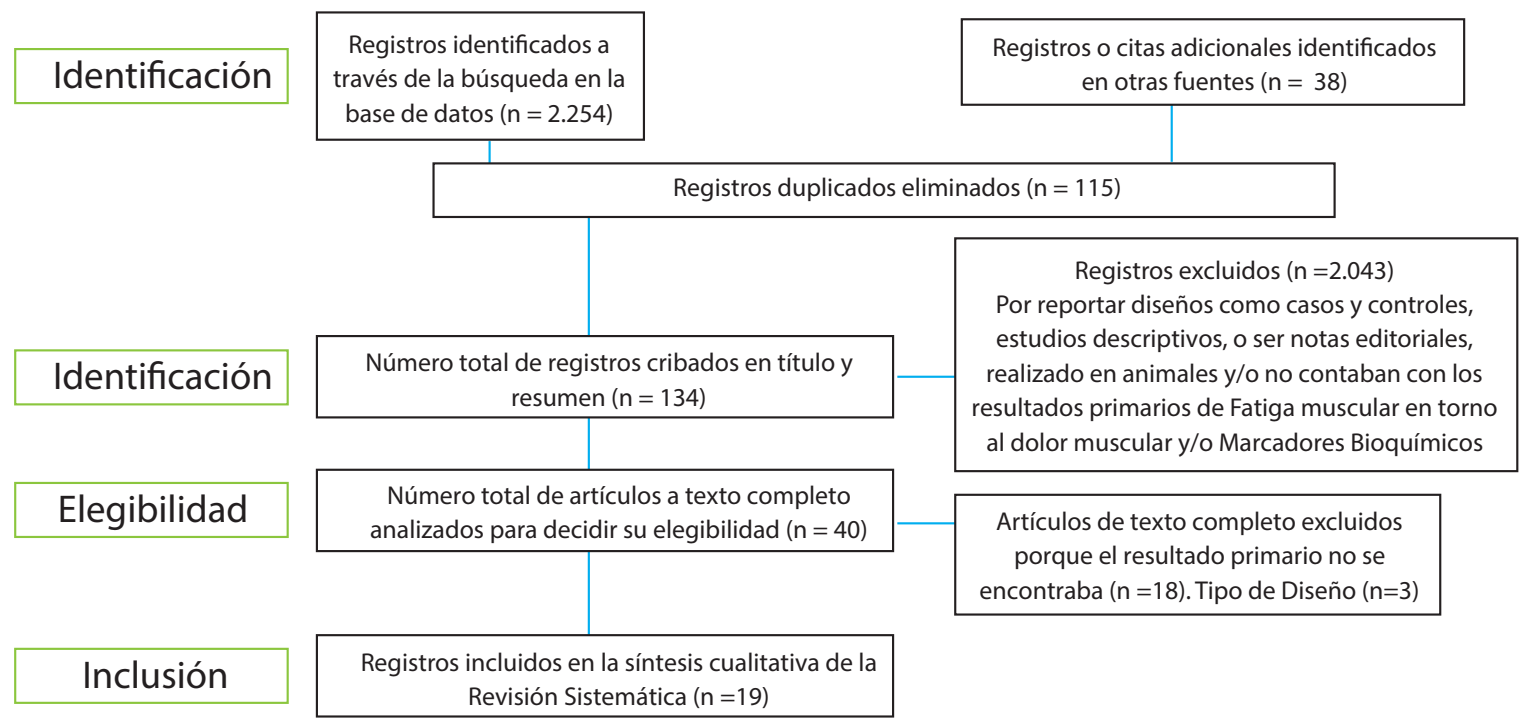

Fuente: Urrútia Gerard BX. Declaración PRISMA: Una propuesta para mejorar la publicación de revisiones sistemáticas y meta análisis. Rev. Medicina Clínica. 2010; 01(15): 507-511

\section{Resultados de la Evaluación del Riesgo de Sesgo y Calidad Metodológica}

La revisión incluyó 19 artículos, en los cuales fue evaluada su calidad metodológica con el uso de la escala PEDro (12), proceso realizado para los ECA que se exponen en la tabla 4.

Atendiendo a que la totalidad de los (ECA) que hacen parte de la presente Revisión Sistemática no fueron encontrados en la Base PEDro, se realizó una evaluación ciega de los ECA restantes con la participación de la investigadora principal, una asesora y un fisioterapeuta externo del proyecto. Con el objeto de validar los resultados se obtuvo el índice de Kappa el cual estableció el grado de concordancia de los puntajes obtenidos entre las evaluadoras, estos índices obtuvieron puntajes entre 0.52 y 1 que, según lo propuesto por Landis (15), serían concordancias clasificadas entre buena y excelente (Tabla 5).

Adicionalmente, en reconocimiento de los usos y limitaciones de la escala de PEDro (16), se realizó la evaluación de riesgo de sesgo basados en los lineamientos del Manual Cochrane de revisiones 
Tabla 3. Características de los estudios incluidos en la revisión sistemática.

\begin{tabular}{|c|c|c|c|c|c|c|c|c|c|c|}
\hline AUTOR - AÑO & $\begin{array}{l}\text { CARACTERÍ́STICAS } \\
\text { DE LA POBLACIÓN }\end{array}$ & CRITERIOS DE & CRITERIOS DE & \multicolumn{2}{|c|}{ INTERVENCIÓN } & \multirow{3}{*}{ 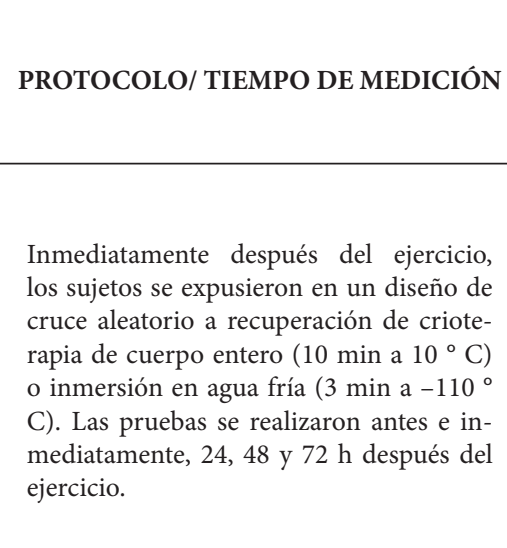 } & \multirow{3}{*}{$\begin{array}{c}\text { DOLOR } \\
\\
\begin{array}{l}\text { Dolor, evalua- } \\
\text { do a través de } \\
\text { Escala Likert }\end{array}\end{array}$} & \multirow{3}{*}{$\begin{array}{c}\text { MARCADORES } \\
\text { BIOQUíMICOS } \\
\\
\\
\text { Actividad } \\
\text { de Creatina } \\
\text { Quinasa. }\end{array}$} & \multirow{3}{*}{$\begin{array}{l}\text { RESULTADOS } \\
\text { SECUNDARIOS } \\
\\
\text { Fuerza excéntrica y } \\
\text { fuera isométrica } \\
\text { evaluada traves de } \\
\text { Dinamómetro isoci- } \\
\text { nético }\end{array}$} & \multirow{3}{*}{ 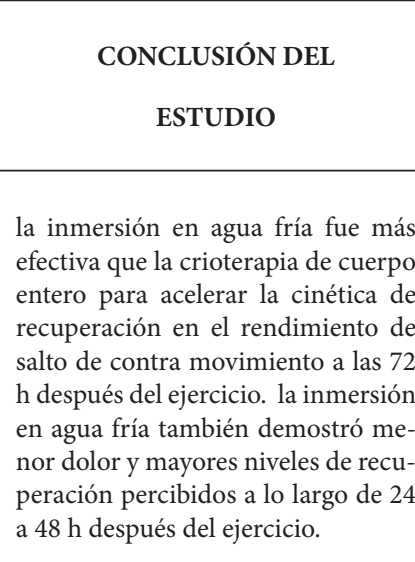 } \\
\hline & & \multirow[b]{2}{*}{$\begin{array}{l}\text { No haber sufrido ninguna } \\
\text { lesion tendinosa en rodila } \\
\text { durante los } 6 \text { meses anteriores. }\end{array}$} & \multirow[b]{2}{*}{ No reporta. } & $\begin{array}{l}\text { GRUPO CONTROL: } \\
\text { n: no refiere }\end{array}$ & $\begin{array}{c}\text { Inmersión en agua } \\
\text { fría }\end{array}$ & & & & & \\
\hline $\begin{array}{l}\text { ABAÏDIA } \\
2017 \\
\text { Francia }\end{array}$ & $\begin{array}{l}10 \text { hombres } \\
\text { fisiscamente activos }\end{array}$ & & & $\begin{array}{c}\text { GRUPO } \\
\text { EXPERIMENTAL: } \\
\text { n: no refiere }\end{array}$ & $\begin{array}{l}\text { Crioterapia de cuerpo } \\
\text { entero }\end{array}$ & & & & & \\
\hline \multirow[b]{2}{*}{$\begin{array}{l}\text { AKLEY } 2013 \\
\text { Estados } \\
\text { Unidos }\end{array}$} & \multirow[b]{2}{*}{$\begin{array}{l}\text { Treinta y tres su- } \\
\text { jetos }\end{array}$} & \multirow{2}{*}{$\begin{array}{l}\text { Se pidió a los sujetos que se } \\
\text { abstuvieran de participar } \\
\text { en cualquier actividad fisica } \\
\text { o cualquier otra forma de } \\
\text { intervención para el tra- } \\
\text { tamiento de los sintomas } \\
\text { dolorososo durante el estudio, } \\
\text { contraindicación médica } \\
\text { fisica al ejercicioio o hielo. }\end{array}$} & \multirow{2}{*}{$\begin{array}{l}\text { Entrenamiento con pesas en } \\
\text { las últimas } 2 \text { semanass cual- } \\
\text { quier reaccion adversaa al } \\
\text { frio, condiciones cardiopul- } \\
\text { monares, problemas en las } \\
\text { articulaciones de extremidad } \\
\text { inferior. }\end{array}$} & $\begin{array}{l}\text { GRUPO CONTROL: } \\
\text { n: } 10\end{array}$ & $\begin{array}{l}\text { No recibió ninguna } \\
\text { intervención }\end{array}$ & \multirow[b]{2}{*}{ 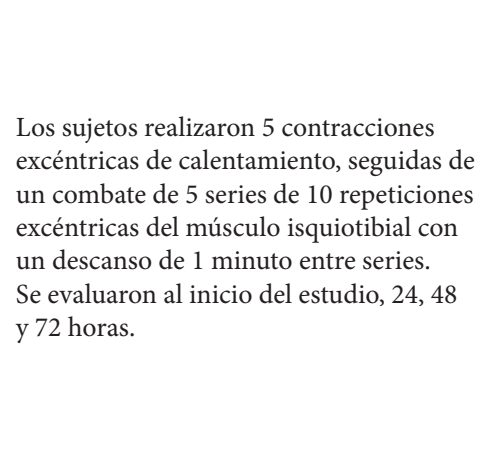 } & \multirow{2}{*}{$\begin{array}{c}\text { Dolor, evaluado } \\
\text { a través de la } \\
\text { Escala Análoga } \\
\text { Visual con } \\
\text { rangos: } \\
\text { 0: sin dolor } \\
\text { 10: peor dolor. }\end{array}$} & \multirow[b]{2}{*}{$\begin{array}{l}\text { CK, ALT, } \\
\text { AST y } \\
\text { neutrófilos. }\end{array}$} & \multirow[b]{2}{*}{$\begin{array}{l}\text { Contracción isomé- } \\
\text { trica voluntaria del } \\
\text { músculo isquiotibial, } \\
\text { evaluada con Dina- } \\
\text { mómetro }\end{array}$} & \multirow[b]{2}{*}{$\begin{array}{l}\text { Las aplicaciones repetidas de hielo } \\
\text { pueden disminuir el dolor asociado } \\
\text { con EIMD significativamente a las } \\
48 \text { horas post EIMD. }\end{array}$} \\
\hline & & & & $\begin{array}{c}\text { GRUPO } \\
\text { EXPERIMENTAL: } \\
\text { n: } 23\end{array}$ & 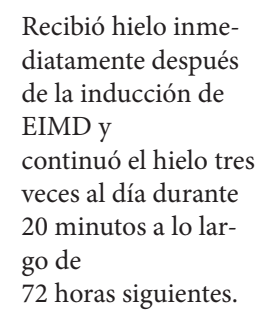 & & & & & \\
\hline \multirow{2}{*}{$\begin{array}{l}\text { ANDAL } 2013 \\
\text { Brasil }\end{array}$} & \multirow{2}{*}{$\begin{array}{c}\text { Diez luchadores } \\
\text { altamente entre- } \\
\text { nados }\end{array}$} & \multirow{2}{*}{$\begin{array}{l}\text { Al menos tres años de expe- } \\
\text { riencia en entrenamiento, y } \\
\text { participación en al menos } \\
\text { tres competencias en el año } \\
\text { anterior a este estudio. }\end{array}$} & \multirow{2}{*}{ No reporta. } & $\begin{array}{l}\text { GRUPO CONTROL: } \\
\text { n: no refiere }\end{array}$ & Sin tratamiento & \multirow{2}{*}{$\begin{array}{l}\text { Inmediatamente después de la última } \\
\text { pelea, los atletas se sumergieron en agua } \\
\text { fría }\left(5 \pm \pm^{\circ} \mathrm{C} \text { C) durante } 19 \text { minutos (cua- }\right. \\
\text { tro ciclos de inmersion de } 4 \text { minutos con } \\
\text { intervalos de } 1 \text { minututo). Durante este } \\
\text { período, los combatientes en el grupo } \\
\text { control permanecieron recostados en } \\
\text { reposo pasivo a temperatura ambiente } \\
\left(26^{\circ} \mathrm{C}\right) \text {. }\end{array}$} & \multirow{2}{*}{$\begin{array}{l}\text { Dolor, evaluado } \\
\text { a través de la } \\
\text { Escala Análoga } \\
\text { Visual }\end{array}$} & \multirow{2}{*}{ No reporta. } & \multirow{2}{*}{$\begin{array}{l}\text { Fuerza de la extremi- } \\
\text { dad superior, evaluado } \\
\text { a través del test Ki- } \\
\text { mono Grip Strength. }\end{array}$} & \multirow{2}{*}{$\begin{array}{l}\text { La inmersión después de la compe- } \\
\text { tencia evidencia mejores resultados } \\
\text { en temperatura corporal, hipoalge- } \\
\text { sia, LDH y CPK, en comparación } \\
\text { con el grupo control. }\end{array}$} \\
\hline & & & & $\begin{array}{c}\text { GRUPO } \\
\text { EXPERIMENTAL: } \\
\text { n: no refiere }\end{array}$ & $\begin{array}{l}\text { Inmersíón en piscina } \\
\text { con hielolo }\end{array}$ & & & & & \\
\hline
\end{tabular}




\begin{tabular}{|c|c|c|c|c|c|c|c|c|c|c|}
\hline $\begin{array}{l}\text { AUTOR - AÑO } \\
\text {-PAÎ́S }\end{array}$ & $\begin{array}{c}\text { CARACTERÍ́STICAS } \\
\text { DE LA POBLACIÓN } \\
\text { ESTUDIO }\end{array}$ & $\begin{array}{l}\text { CRITERIOS DE } \\
\text { INCLUSIÓN }\end{array}$ & $\begin{array}{l}\text { CRITERIOS DE } \\
\text { EXCLUSIÓN }\end{array}$ & INTER & NCIÓN & PROTOCOLO/ TIEMPO DE MEDICIÓN & DOLOR & $\begin{array}{l}\text { MARCADORES } \\
\text { BIOQUIMIICOS }\end{array}$ & $\begin{array}{l}\text { RESULTADOS } \\
\text { SECUNDARIOS }\end{array}$ & $\begin{array}{l}\text { CONCLUSIÓN DEL } \\
\text { ESTUDIO }\end{array}$ \\
\hline $\begin{array}{l}\text { ASCENSAO } \\
\text { 2011 } \\
\text { Portugal }\end{array}$ & $\begin{array}{l}20 \text { futbolistas } \\
\text { juveniles mascu- } \\
\text { linos }\end{array}$ & $\begin{array}{l}\text { Jugadores del equipo que } \\
\text { sean defensores, mediocam- } \\
\text { pistas, ylos delanteros. }\end{array}$ & $\begin{array}{l}\text { Porteros fueron excluidos de } \\
\text { la investigación. }\end{array}$ & $\begin{array}{c}\text { GRUPO CONTROL: } \\
\text { n: no refiere } \\
\text { GRUPO } \\
\begin{array}{c}\text { EXPERIMENTAL: } \\
\text { n: no refiere }\end{array}\end{array}$ & Agua termoneural & 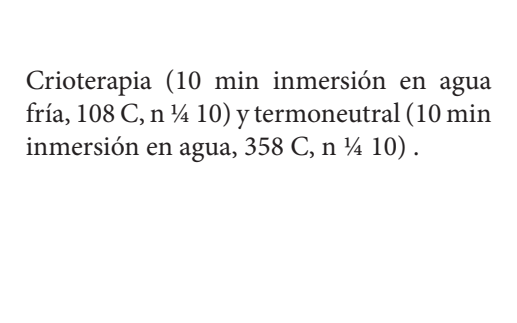 & $\begin{array}{l}\text { Dolor, evaluado } \\
\text { a través del } \\
\text { Cuestionario de } \\
\text { dolor muscular }\end{array}$ & $\begin{array}{l}\text { Actividad de } \\
\text { la creatina } \\
\text { quinasa plas- } \\
\text { mática, con- } \\
\text { centraciones } \\
\text { de mioglobina } \\
\text { y proténa C } \\
\text { reactiva }\end{array}$ & No reporta. & $\begin{array}{l}\text { Los resultados sugieren que la in- } \\
\text { mersión en agua fría inmediata- } \\
\text { mente después de un partido de } \\
\text { fútbol único reduce el daño mus- } \\
\text { cular y malestar, posiblemente con- } \\
\text { tribuyendo a una recuperación más } \\
\text { rápida de la función neuromuscular. }\end{array}$ \\
\hline $\begin{array}{l}\text { BAILEY } 2017 \\
\text { Reino Unido }\end{array}$ & 20 hombres sanos & $\begin{array}{l}\text { Ser habitualmente activos } \\
\text { en una variedad de deportes } \\
\text { y que se pudieran abstener } \\
\text { de tratamientos terapeuticos } \\
\text { que incluyeran masajes y } \\
\text { medicamentos antinfflama- } \\
\text { torios durante la duración } \\
\text { de la investigación. }\end{array}$ & No reporta. & $\begin{array}{c}\begin{array}{c}\text { GRUPO CONTROL: } \\
\text { n: no refiere }\end{array} \\
\\
\text { GRUPO } \\
\text { EXPERIMENTAL: } \\
\text { n: no refiere }\end{array}$ & Sin tratamiento & $\begin{array}{l}\text { El ejercicio resultó en dolor muscular } \\
\text { intenso, disfunción muscular temporal y } \\
\text { marcadores séricos elevados de daño mus- } \\
\text { cular, que alcanzzaron su punto máximo } \\
\text { dentro de las } 48 \text { h posteriorese al ejercicio. } \\
\text { La crioterapia administrada inmediata- } \\
\text { mentedespués del ejercicio redujo el dolor } \\
\text { muscular a las } 1,24 \text { y } 48 \text { h. }\end{array}$ & $\begin{array}{l}\text { Dolor, evaluado } \\
\text { a través de la } \\
\text { Escala analógi- } \\
\text { ca visual }\end{array}$ & $\begin{array}{l}\text { Actividad de } \\
\text { creatina qui- } \\
\text { nasa. (CK) }\end{array}$ & $\begin{array}{l}\text { Máxima contracción } \\
\text { isométrica voluntaria } \\
\text { (MVC), evaluado a } \\
\text { través de Dinamóme- } \\
\text { tro isocinético (Cybex } \\
\text { modelo 770, LUMEX } \\
\text { Inc., Ronkonkoma, EE. } \\
\text { UU.). }\end{array}$ & 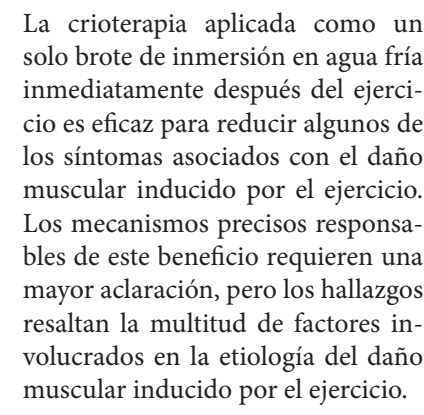 \\
\hline $\begin{array}{l}\text { BEHRINGER } \\
2018 \\
\text { Alemania }\end{array}$ & $\begin{array}{c}30 \text { estudiantes de } \\
\text { deportes saludables } \\
\text { (21 hombres, } 9 \\
\text { mujeres) }\end{array}$ & No reporta. & $\begin{array}{l}\text { Cualquier enfermedad car- } \\
\text { diovascular, metabólica y } \\
\text { lesiones del sistema muscu- } \\
\text { loosquelético dentro de los } 6 \\
\text { meses anteriores al estudio. }\end{array}$ & $\begin{array}{c}\text { GRUPO CONTROL: } \\
\text { n: no refiere } \\
\\
\text { GRUPO } \\
\text { EXPERIMENTAL: } \\
\text { n: no refiere }\end{array}$ & $\begin{array}{l}\text { Drenaje linfítico } \\
\text { manual (MLD) } \\
\text { (30 muntos) } \\
\text { Crioterapia local } \\
\text { (CRY) (30 minutos) }\end{array}$ & $\begin{array}{l}\text { Después de } 4 x 20 \text { extensiones de rodilla } \\
\text { acentuadasas excéntricasy ynilaterales (con } \\
\text { un tercio de la fuerza isométrica máxima), } \\
\text { los participantes recibieron al azar drenaje } \\
\text { linfático, crioterapia local o descanso (30 } \\
\text { minutos cada uno). }\end{array}$ & $\begin{array}{l}\text { Dolor, evaluado } \\
\text { a través de la } \\
\text { Escala analógi- } \\
\text { ca visual }\end{array}$ & $\begin{array}{l}\text { Concentra- } \\
\text { ción de crea- } \\
\text { tina quinasa } \\
\text { CK Proténa } \\
\text { de unióna } \\
\text { aćidos grasos } \\
\text { (h-FA BP), } \\
\text { número de } \\
\text { granulocitos } \\
\text { nuutrófilos }\end{array}$ & $\begin{array}{l}\text { Fuerza isométrica } \\
\text { máxima. Evaluada a } \\
\text { través de la máquina } \\
\text { de extensión de pier- } \\
\text { nas (Gym } 80 \text { - Edition } \\
\text { Line, Gymm } 8 \text { Interna- } \\
\text { tional, Gelsenkirchen, } \\
\text { Alemania) }\end{array}$ & 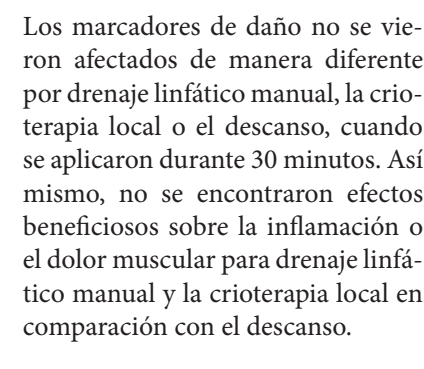 \\
\hline
\end{tabular}




\begin{tabular}{|c|c|c|c|c|c|c|c|c|c|c|}
\hline $\begin{array}{l}\text { AUTOR - AÑo } \\
\text {-PAî́S }\end{array}$ & $\begin{array}{c}\text { CARACTERÍSTICAS } \\
\text { DE LA POBLACIÓN } \\
\text { ESTUDIO }\end{array}$ & $\begin{array}{l}\text { CRITERIOS DE } \\
\text { INCLUSIÓN }\end{array}$ & $\begin{array}{l}\text { CRITERIOS DE } \\
\text { EXCLUSIÓN }\end{array}$ & INTER & NCIÓN & PROTOCOLO/ TIEMPO DE MEDICIÓN & DOLOR & $\begin{array}{l}\text { MARCADORES } \\
\text { BIOQUÍMICOS }\end{array}$ & $\begin{array}{l}\text { RESULTADOS } \\
\text { SECUNDARIOS }\end{array}$ & $\begin{array}{c}\text { CONCLUSIÓN DEL } \\
\text { ESTUDIO }\end{array}$ \\
\hline $\begin{array}{l}\text { CRYSTAL } \\
\mathbf{2 0 1 3} \\
\text { Estados } \\
\text { Unidos }\end{array}$ & 20 hombres activos & $\begin{array}{l}\text { Hombres que no estaban acos- } \\
\text { tumbrados a la crioterapia. }\end{array}$ & 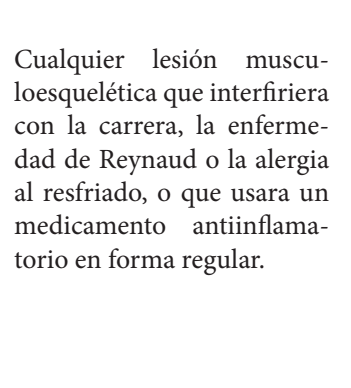 & $\begin{array}{l}\text { GRUPO } \\
\text { EXPERIMENTAL: } \\
\text { n: no refiere }\end{array}$ & 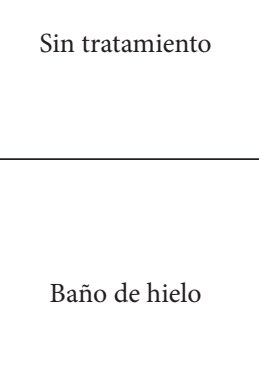 & $\begin{array}{l}\text { Se sumergieron en un baño de hielo a } 5^{\circ} \mathrm{C} \\
\text { durante } 20 \text { minutos. }\end{array}$ & $\begin{array}{l}\text { Dolor. Evalua- } \\
\text { da con Escala } \\
\text { analógica visual }\end{array}$ & $\begin{array}{l}\text { Citoquina } \\
\text { inflamatoria } \\
\text { CCL2 }\end{array}$ & $\begin{array}{l}\text { Fuerza isométrica, } \\
\text { evaluada a través de } \\
\text { Dinamomónetro isoci- } \\
\text { nético. } \\
\text { Inflamación, evaluada } \\
\text { con Cinta métrica } \\
\text { antropométrica }\end{array}$ & 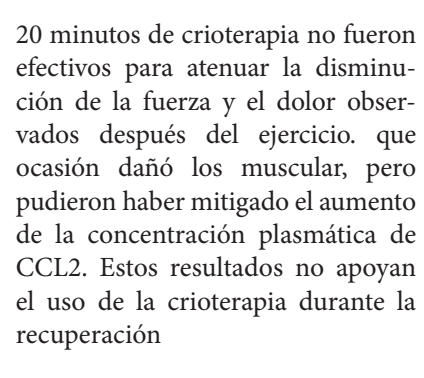 \\
\hline $\begin{array}{l}\text { DEMARCHI } \\
\text { 2017 } \\
\text { Brasil }\end{array}$ & $\begin{array}{l}40 \text { individuos de } 19 \\
\text { a } 29 \text { años }\end{array}$ & No reporta. & $\begin{array}{l}\text { Lesiones musculoesquelé- } \\
\text { ticas } 3 \text { meses anteriores a la } \\
\text { investigación, uuso de suple- } \\
\text { mentos nutriciconales of far- } \\
\text { macológicos. }\end{array}$ & 5 grupos & $\begin{array}{c}\text { Grupo } \\
\text { fotobiomodulación } \\
\text { PBMT } \\
\end{array}$ & $\begin{array}{l}4 \text { sesiones con intervalos de } 24 \text { horas. El } \\
\text { primer dia fueron sometidos a protoco- } \\
\text { lo inductor de fatiga muscular (MFII), y } \\
\text { extracción de sangre durante el periodo } \\
\text { previo al ejercicio, } 5 \text { minutos y } 60 \text { min des- } \\
\text { pués. En las demás sesiones realizadas a } \\
\text { las } 24,48 \text { y } 72 \text { horas posteriores se realizó } \\
\text { recolección de sangrey evaluación isomé- } \\
\text { trica (MVC) en el dinamámetro cinético. }\end{array}$ & $\begin{array}{l}\text { Dolor, evaluado } \\
\text { a través de la } \\
\text { Escala Análoga } \\
\text { Visual. }\end{array}$ & $\begin{array}{l}\text { Concentra- } \\
\text { ciones de la } \\
\text { bioquímica. } \\
\text { marcador de } \\
\text { daño muscular } \\
\text { (CK) }\end{array}$ & $\begin{array}{l}\text { Masa Corporal eva- } \\
\text { luada en (Kg). } \\
\text { Contracción iso- } \\
\text { métrica, evaluada } \\
\text { con Dinamómetro } \\
\text { cinético. }\end{array}$ & 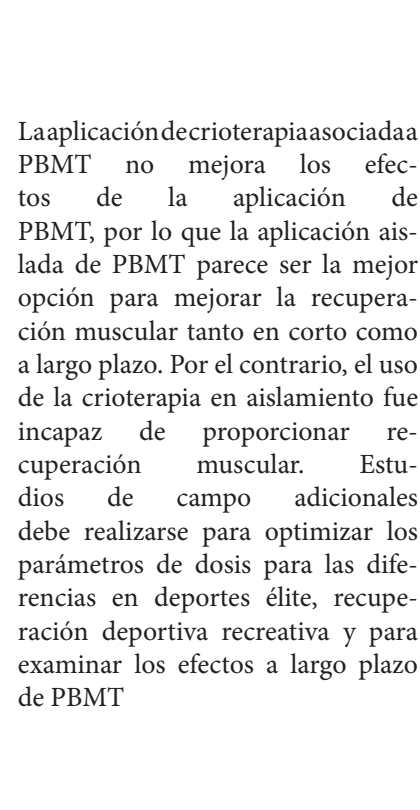 \\
\hline
\end{tabular}




\begin{tabular}{|c|c|c|c|c|c|c|c|c|c|c|}
\hline $\begin{array}{l}\text { AUTOR - AÑO } \\
\text {-PAÎ́S }\end{array}$ & $\begin{array}{c}\text { CARACTERÍSTICAS } \\
\text { DE LA POBLACIÓN } \\
\text { ESTUDIO }\end{array}$ & $\begin{array}{l}\text { CRITERIOS DE } \\
\text { INCLUSIÓN }\end{array}$ & $\begin{array}{l}\text { CRITERIOS DE } \\
\text { EXCLUSIÓN }\end{array}$ & INTER & ENCIÓN & PROTOCOLO/ TIEMPO DE MEDICIÓN & DOLOR & $\begin{array}{l}\text { MARCADORES } \\
\text { BIOQUÍMICOS }\end{array}$ & $\begin{array}{l}\text { RESULTADOS } \\
\text { SECUNDARIOS }\end{array}$ & $\begin{array}{l}\text { CONCLUSIÓN DEL } \\
\text { ESTUDIO }\end{array}$ \\
\hline $\begin{array}{l}\text { DEPAIVA } \\
2016 \\
\text { Londres }\end{array}$ & $\begin{array}{l}\text { Cincuenta } \\
\text { sujetos sanos no } \\
\text { entrenados de la } \\
\text { universidad. }\end{array}$ & 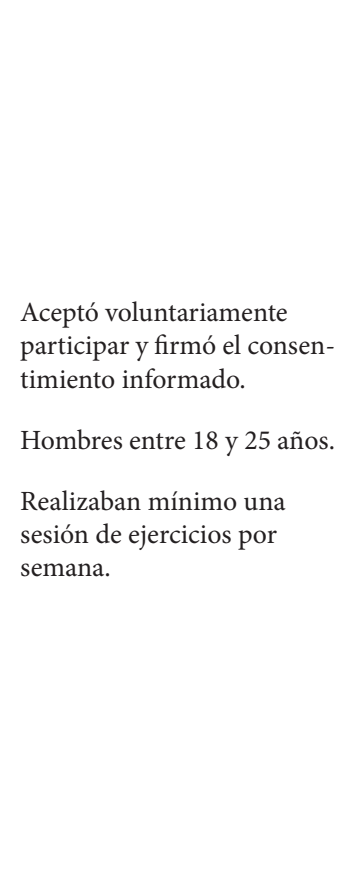 & $\begin{array}{l}\text { Lesión musculoesquelética } \\
\text { en las caderas o rodilias den- } \\
\text { trode los } 2 \text { meses anterioreres, } \\
\text { uso de fermacos, agentes } \\
\text { suplementos nutricionales } \\
\text { con regularidad y consumo } \\
\text { de alcoholo otabaco. }\end{array}$ & $\begin{array}{l}5 \text { Grupos: } 10 \text { por } \\
\text { grupo. }\end{array}$ & $\begin{array}{c}\text { Placebo } \\
\text { PBMT (fótobiomodu- } \\
\text { lación) } \\
- \\
\text { Crioterapia }\end{array}$ & 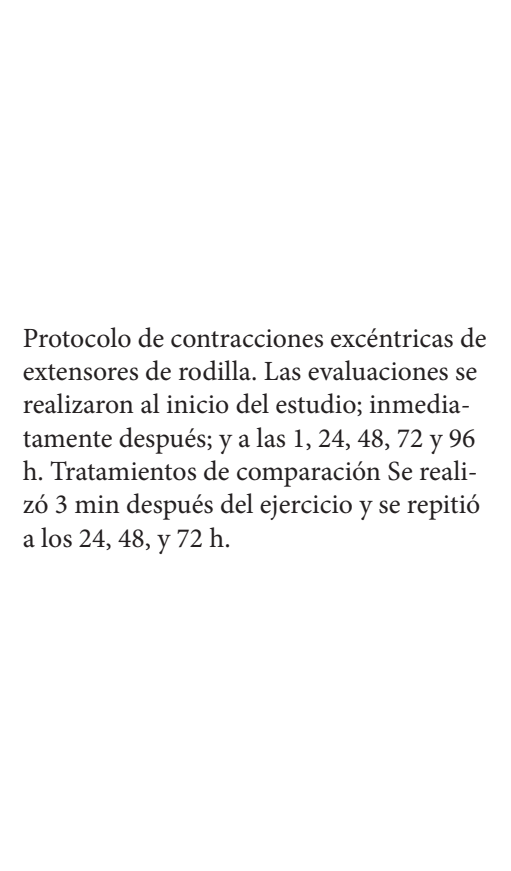 & $\begin{array}{l}\text { DOMS Do- } \\
\text { lor muscular } \\
\text { Escala análoga } \\
\text { visual }\end{array}$ & $\begin{array}{l}\text { Creatina Qui- } \\
\text { nasa (CK) }\end{array}$ & $\begin{array}{l}\text { MVC Méxima contrac. } \\
\text { ción voluntaria }\end{array}$ & 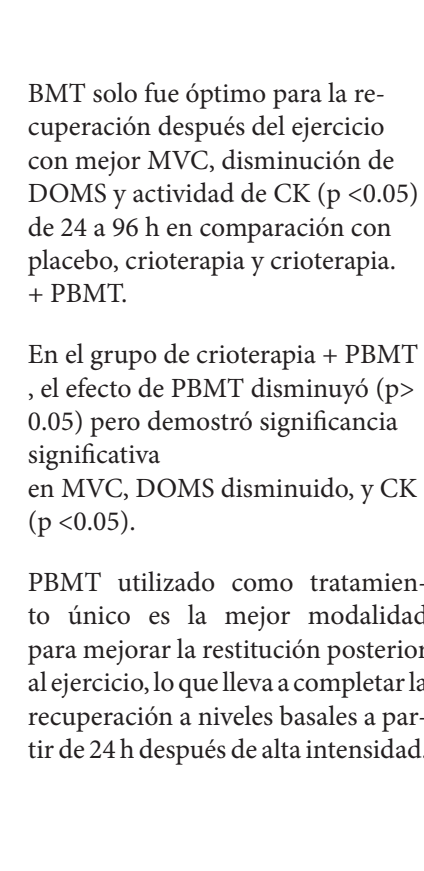 \\
\hline $\begin{array}{l}\text { FONSECA } \\
2016 \\
\text { Brasil }\end{array}$ & $\begin{array}{l}8 \text { atletas mascu- } \\
\text { linos }\end{array}$ & $\begin{array}{l}\text { Atletas con graduación } \\
\text { como cinturón azul o púr- } \\
\text { pura, participación en al } \\
\text { menos } 3 \text { competiciones en } \\
\text { el año anterior al estudioy } \\
\text { no participar en cualquier } \\
\text { proceso rápido de pérdida } \\
\text { de peso antes de la compe- } \\
\text { tencia. }\end{array}$ & $\begin{array}{l}\text { No cumplir con los criterios } \\
\text { de inclusión. }\end{array}$ & $\begin{array}{c}\text { GRUPO CONTROL: } \\
\text { n: no refiere } \\
\\
\text { GRUPO } \\
\text { EXPERIMENTAL: } \\
\text { n: no refiere }\end{array}$ & $\begin{array}{l}\text { Inmersión en agua } \\
\text { fría (CWI) }\end{array}$ & $\begin{array}{l}\text { Inmersión en agua fría (CWI) }(6.08 \mathrm{C} 6 \\
0.58 \mathrm{C}) \text { durante } 19 \text { minutos. }\end{array}$ & $\begin{array}{l}\text { Dolor, Evalua- } \\
\text { da con Escala } \\
\text { análoga visual }\end{array}$ & $\begin{array}{l}\text { Concentra- } \\
\text { ciones séricas } \\
\text { de CK, LDH, } \\
\text { AST y ALT }\end{array}$ & $\begin{array}{l}\text { Masa corporal, eva- } \\
\text { luada en }(\mathrm{Kg})\end{array}$ & $\begin{array}{l}\text { El uso de inmersión en agua fría } \\
\text { (CWI) puede ser beneficioso para } \\
\text { los attetas de jij-jitsu porqueu reduce } \\
\text { los niveles circulantes de LDH, pro- } \\
\text { duce menos dolor muscular percibi- } \\
\text { do y ayuda a recuperar la potencia } \\
\text { muscular } 24 \text { horas después de la } \\
\text { recuperación. }\end{array}$ \\
\hline
\end{tabular}




\begin{tabular}{|c|c|c|c|c|c|c|c|c|c|c|}
\hline $\begin{array}{l}\text { AUTOR - AÑo } \\
\text {-PAÍS }\end{array}$ & $\begin{array}{c}\text { CARACTERÍ́STICAS } \\
\text { DE LA POBLACIÓN } \\
\text { ESTUDIO }\end{array}$ & $\begin{array}{l}\text { CRITERIOS DE } \\
\text { INCLUSIÓN }\end{array}$ & $\begin{array}{l}\text { CRITERIOS DE } \\
\text { EXCLUSIÓN }\end{array}$ & INTEI & NCIÓN & PROTOCOLO/ TIEMPO DE MEDICIÓN & DOLOR & $\begin{array}{l}\text { MARCADORES } \\
\text { BIOQUÍMICOS }\end{array}$ & $\begin{array}{l}\text { RESULTADOS } \\
\text { SECUNDARIOS }\end{array}$ & $\begin{array}{l}\text { CONCLUSIÓN DEL } \\
\text { ESTUDIO }\end{array}$ \\
\hline $\begin{array}{l}\text { GOODALL } \\
2008 \\
\text { Reino Unido }\end{array}$ & $\begin{array}{l}18 \text { hombres fisica- } \\
\text { mente activos }\end{array}$ & $\begin{array}{l}\text { Completar un cuestionario } \\
\text { de salud médica y propor- } \\
\text { cionar consentimiento in- } \\
\text { formado por escrito. }\end{array}$ & No reporta. & $\begin{array}{c}\text { GRUPO CONTROL: } \\
\mathrm{n}: 9 \\
\end{array}$ & Sin tratamiento & $\begin{array}{l}\text { Inmersiones en agua fría (CWI) }(15 \pm 1 \\
{ }^{\circ} \mathrm{C} \text { ) que experimentaron inmersiones in- } \\
\text { medidatamente después del jerrcici y cada } \\
24 \text { h posteriores durante los siguientes } 3 \\
\text { dias. } \\
\text { Grupo de control (sin tratamiento). }\end{array}$ & $\begin{array}{l}\text { Dolor, evalua- } \\
\text { do con Escala } \\
\text { Análoga visual }\end{array}$ & $\begin{array}{l}\text { Actividad de } \\
\text { la creatina } \\
\text { quinasa plas- } \\
\text { mática (CK) }\end{array}$ & 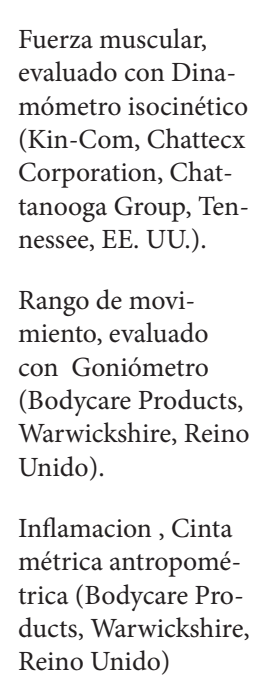 & 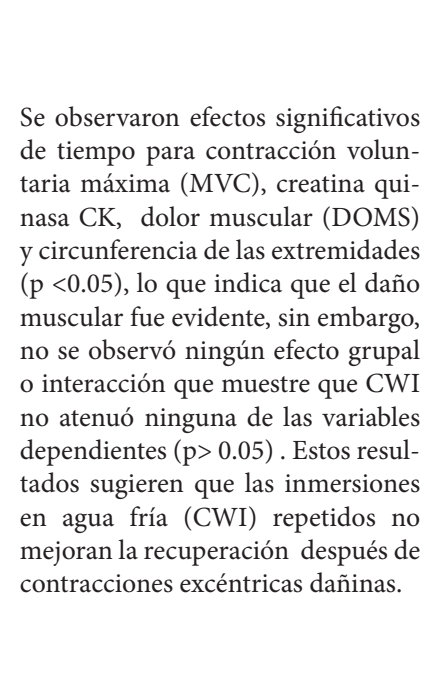 \\
\hline $\begin{array}{l}\text { HOWATSON } \\
\text { 2005 } \\
\text { Londres }\end{array}$ & 12 hombres & $\begin{array}{l}\text { Consentimiento informado, } \\
\text { abstenerse de actividades } \\
\text { que puedan causar dano, } \\
\text { dolor y, por lo tanto, influir } \\
\text { en las varialles dependien- } \\
\text { tes durante la duración de la } \\
\text { investigación. }\end{array}$ & No reporta. & $\begin{array}{c}\text { GRUPO CONTROL: } \\
\text { n: no refiere } \\
\text { GRUPO } \\
\text { EXPERIMENTAL: } \\
\text { n: no refiere }\end{array}$ & Ecografía simulada & $\begin{array}{l}\text { El protocolo constaba de tres series de } 10 \\
\text { repeticiones excéntricas máximas de los } \\
\text { flexores del codo utilizando dinamome- } \\
\text { tría isocinética. Se evaluó inmediatamente } \\
\text { después del eercicioo, } 24,48,72 \text { y } 96 \mathrm{~h} \text { des- } \\
\text { pués del ejercicicio. }\end{array}$ & $\begin{array}{l}\text { DOMS. Eva- } \\
\text { luado atraves } \\
\text { lde la Escala } \\
\text { Talag on } \\
\text { rangos: } \\
\text { 1: sin dolor } \\
\text { 7: insoporta- } \\
\text { blemente do- } \\
\text { loroso }\end{array}$ & $\mathrm{CK}, \mathrm{Mb}$. & No refiere & $\begin{array}{l}\text { El masaje con hielo es ineficaz para } \\
\text { reducir los marcadores indirectos } \\
\text { asociados con el dano muscular } \\
\text { inducido por el ejercicio y ypar me- } \\
\text { jorar la recuperación de la función } \\
\text { muscular en los deportistas mascu- } \\
\text { linos no acostumbrados al ejercicio } \\
\text { sesgado excéntrico. }\end{array}$ \\
\hline $\begin{array}{l}\text { JAKEMAN } \\
2019 \\
\text { Reino Unido }\end{array}$ & 18 mujeres atletas & $\begin{array}{l}\text { Tener lesiones musculares o } \\
\text { esqueléticicas antes del la par- } \\
\text { ticipacion, firmar un con- } \\
\text { sentimiento informado. }\end{array}$ & $\begin{array}{l}\text { Haber realizado ningún } \\
\text { entrenamiento pliométrico } \\
\text { especifico o de extremida- } \\
\text { des inferiores en al menos } 6 \\
\text { semanas antes de la prueba. }\end{array}$ & $\begin{array}{c}\begin{array}{c}\text { GRUPO CONTROL: } \\
\text { n: no refiere }\end{array} \\
\text { GRUPO E } \\
\text { XPERIMENTAL: : } \\
\text { no refiere }\end{array}$ & $\begin{array}{c}\text { Inmersión en agua } \\
\text { fría }\end{array}$ & $\begin{array}{l}\text { Inmersión en agua fría con extremidades } \\
\text { inferiores durante } 10 \text { minutos a } 108^{\circ} \mathrm{C} \\
\text { inmediatamente después del ejercicio in- } \\
\text { ductor de daño. }\end{array}$ & $\begin{array}{l}\text { Dolor, evaluado } \\
\text { a través de la } \\
\text { Escala análoga } \\
\text { visual }\end{array}$ & $\begin{array}{c}\text { Actividad } \\
\text { de creatina } \\
\text { quinasa } \\
\text { CK) }\end{array}$ & $\begin{array}{l}\text { Fuerza muscular, } \\
\text { evaluada con Dinamó- } \\
\text { metro isocinético }\end{array}$ & $\begin{array}{l}\text { Los resultados indican que un solo } \\
\text { episodio de inmersión en agua arría } \\
\text { después de un ejercicio dañino no } \\
\text { tiene efectos beneficiosos en la re- } \\
\text { cuperación. }\end{array}$ \\
\hline
\end{tabular}




\begin{tabular}{|c|c|c|c|c|c|c|c|c|c|c|}
\hline $\begin{array}{c}\text { AUTOR - AÑo } \\
\text {-PAÎ́S }\end{array}$ & $\begin{array}{c}\text { CARACTERÍ́STICAS } \\
\text { DE LA POBLACIÓN } \\
\text { ESTUDIO }\end{array}$ & $\begin{array}{l}\text { CRITERIOS DE } \\
\text { INCLUSIÓN }\end{array}$ & $\begin{array}{l}\text { CRITERIOS DE } \\
\text { EXCLUSIÓN }\end{array}$ & INTERI & ENCIÓN & PROTOCOLO/ TIEMPO DE MEDICIÓN & DOLOR & $\begin{array}{l}\text { MARCADORES } \\
\text { BIOQUIMIICOS }\end{array}$ & $\begin{array}{l}\text { RESULTADOS } \\
\text { SECUNDARIOS }\end{array}$ & $\begin{array}{l}\text { CONCLUSIÓN DEL } \\
\text { ESTUDIO }\end{array}$ \\
\hline $\begin{array}{c}\text { MISSAU } \\
2018 \\
\text { Brasil }\end{array}$ & $\begin{array}{l}13 \text { voluntarios } \\
\text { sanos entre 20 y } 35 \\
\text { años con un indice } \\
\text { de masa corporal } \\
\text { (IMC) <30, prac- } \\
\text { tiacaban actividad } \\
\text { fisica y / o hacian } \\
\text { ejercicic con regu- } \\
\text { laridad (+n veg por } \\
\text { semana) }\end{array}$ & $\begin{array}{l}\text { No estar participando en } \\
\text { programas de dieta y no } \\
\text { tener diagnóstito previo } \\
\text { de enfermedades reumato- } \\
\text { logicas, cardiovaculares, } \\
\text { metaboblicas, neurologicas, } \\
\text { oncológicas, inmunologicas } \\
\text { o hematologicass. }\end{array}$ & 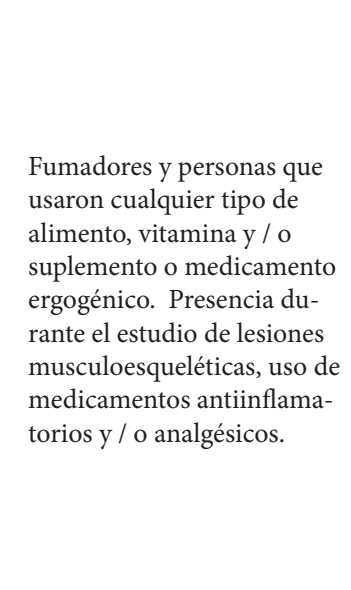 & $\begin{array}{c}\text { GRUPO CONTROL: } \\
\text { n: no refiere } \\
\\
\text { GRUPO } \\
\substack{\text { EXPERIMENTAL: } \\
\text { n: no refiere }}\end{array}$ & $\begin{array}{l}\text { Ejercicios resistidos } \\
\end{array}$ & 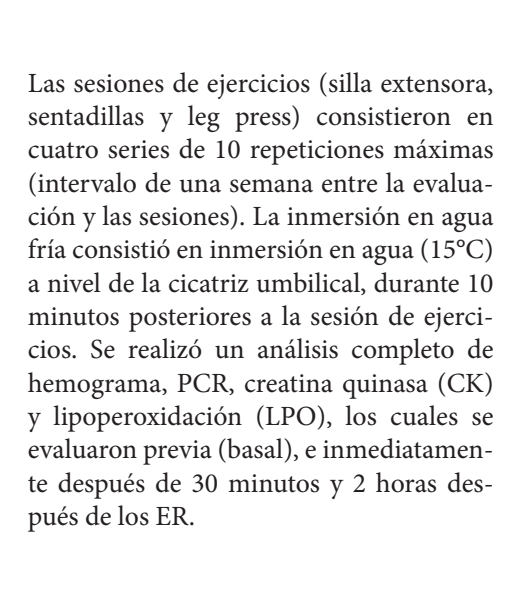 & $\begin{array}{l}\text { Dolor Muscu- } \\
\text { lar Residual, } \\
\text { evaluadoa a } \\
\text { través de la } \\
\text { Escala Análoga } \\
\text { Visual }\end{array}$ & 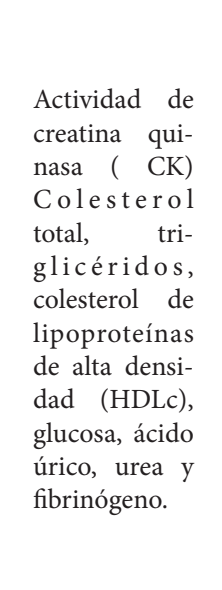 & $\begin{array}{l}\text { Fuerza máxima, eva- } \\
\text { luada con la prueba } \\
10 \mathrm{RM}\end{array}$ & 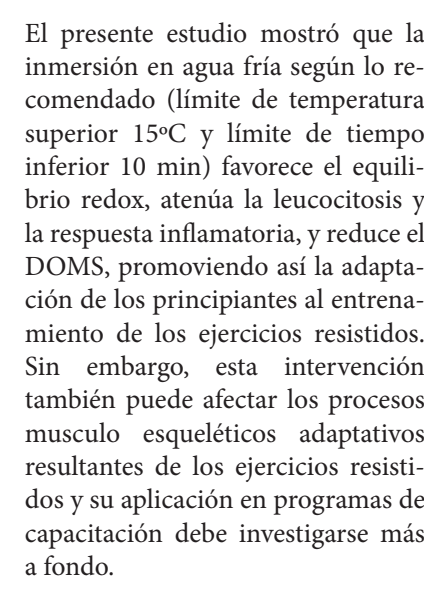 \\
\hline $\begin{array}{l}\text { POINTON } \\
2011 \\
\text { Australia }\end{array}$ & $\begin{array}{l}\text { Diez atletas mas- } \\
\text { culinos entrenados } \\
\text { en resistencia } 21 \\
\pm 1.6 \text { años, altura } \\
182.2 \pm 3.6 \mathrm{~cm} y \\
\text { masa corporal } 87.3 \\
\pm 9.3 \mathrm{~kg}\end{array}$ & No reporta. & No reporta. & $\begin{array}{l} \\
\text { GRUPO } \\
\text { EXPERIMENTAL: } \\
\text { n: no refiere }\end{array}$ & $\begin{array}{l}\text { Hielo cubriendo toda } \\
\text { la superficie de la } \\
\text { Pierna (cuadricess, } \\
\text { rodilla y yantorrilla) } \\
\text { temperatura del agua } \\
=0.5^{\circ} \text { C. durante } 20 \\
\text { minutos. }\end{array}$ & 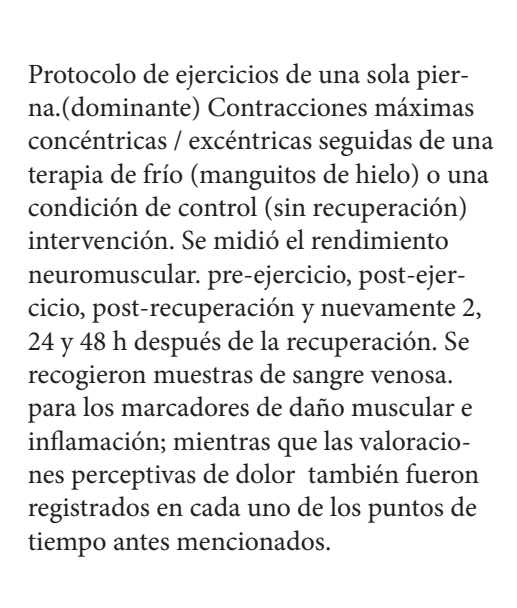 & $\begin{array}{l}\text { Dolor, se eva- } \\
\text { luó con el test } \\
\text { Algometros ain } \\
\text { TestTM FPI } \\
\text { Algometer, } \\
\text { Wagner Ins- } \\
\text { truments según } \\
\text { los siguientes } \\
\text { rangos: } \\
0=\text { sin dolor } \\
\text { y10 muy, } \\
\text { muy adolorido, } \\
\text { Greenwich, } \\
\text { CT, EE. UU. El } \\
\text { valor se regis- } \\
\text { tró en kg } / \mathrm{f} \text { f: } \\
\text { fuerza }\end{array}$ & $\begin{array}{l}\text { Creatina Qui- } \\
\text { nasa (CK), } \\
\text { proteína C } \\
\text { reactiva. } \\
\text { (CRP) y } \\
\text { aspartato ami- } \\
\text { notransferasa } \\
\text { (AST). }\end{array}$ & No reporta. & $\begin{array}{l}\text { No se evidenció ningún efecto } \\
\text { beneficiooso de RRíO en la recupe- } \\
\text { ración de MVC, se redujeron las } \\
\text { percepciones de dolor. }\end{array}$ \\
\hline
\end{tabular}




\begin{tabular}{|c|c|c|c|c|c|c|c|c|c|c|}
\hline $\begin{array}{l}\text { AUTOR - AÑO } \\
\text {-PAİS }\end{array}$ & $\begin{array}{l}\text { CARACTERÍ́STICAS } \\
\text { DE LA POBLACIÓN } \\
\text { ESTUDIO }\end{array}$ & $\begin{array}{l}\text { CRITERIOS DE } \\
\text { INCLUSIÓN }\end{array}$ & $\begin{array}{l}\text { CRITERIOS DE } \\
\text { EXCLUSIÓN }\end{array}$ & INTER & ENCIÓN & PROTOCOLO/ TIEMPO DE MEDICIÓN & DOLOR & $\begin{array}{l}\text { MARCADORES } \\
\text { BIOQUIMIICOS }\end{array}$ & $\begin{array}{l}\text { RESULTADOS } \\
\text { SECUNDARIOS }\end{array}$ & $\begin{array}{l}\text { CONCLUSIÓN DEL } \\
\text { ESTUDIO }\end{array}$ \\
\hline $\begin{array}{l}\text { ROWSEL } \\
2009 \\
\text { Australia }\end{array}$ & $\begin{array}{l}\text { Veinte jugadores } \\
\text { juveniles de fútbol } \\
\text { masculino de alto } \\
\text { rendimieinto (edad } \\
15.9+0.6 \text { años) }\end{array}$ & $\begin{array}{l}\text { No consumir antiinflama- } \\
\text { torios, medicamentos y } \\
\text { suplementos nutricionales. } \\
\text { Sin presencia de lesiones y } \\
\text { enfermedades conocidas al } \\
\text { inicio del estudio. }\end{array}$ & $\begin{array}{l}\text { Porteros fueron excluidos de } \\
\text { la investigación. }\end{array}$ & $\begin{array}{c}\text { GRUPO CONTROL: } \\
\text { n: no refiere } \\
\text { GRUPO } \\
\text { EXPERIMENTAL: } \\
\text { n: no refiere }\end{array}$ & $\begin{array}{c}\begin{array}{c}\text { Inmersión en agua } \\
\text { fría } 10+0.58 \mathrm{C} C \\
\text { durante } 20 \text { minutos. }\end{array} \\
\begin{array}{l}\text { Inmersión en agua } \\
\text { termoneural } 34+ \\
0,58 C \text { C. durante } 20 \\
\text { minutos. }\end{array}\end{array}$ & 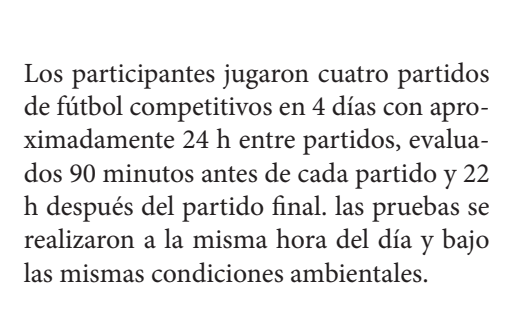 & $\begin{array}{l}\text { Dolor Escala } \\
\text { de } 1 \text { a } 10,24 \\
\text { h después de } \\
\text { cada partido. }\end{array}$ & $\begin{array}{l}\text { Protéína de } \\
\text { unión a ácidos } \\
\text { grasos, lactato } \\
\text { deshidroge- } \\
\text { nasa, plasma, } \\
\text { actividad de } \\
\text { la creatina } \\
\text { quinasa CK. }\end{array}$ & No reporta. & $\begin{array}{l}\text { Estos resultados sugieren que la } \\
\text { inmersión inmediata en agua fría } \\
\text { posterior al partido no afectael ren- } \\
\text { dimiento de la prueba fisica o indi- } \\
\text { ces de daño musculare e inflamación, } \\
\text { pero reduce la percepción de fatiga } \\
\text { general y dolor en las piernas entre } \\
\text { partidos en torneos. }\end{array}$ \\
\hline $\begin{array}{c}\text { TSENG } 2013 \\
\text { China }\end{array}$ & $\begin{array}{l}11 \text { sujetos masculi- } \\
\text { nos de béisbol }\end{array}$ & $\begin{array}{l}\text { No haber entrenado con pe- } \\
\text { sas in ninguna otra forma } \\
\text { de entrenamiento con ejer- } \\
\text { cicios durante al menos } 1 \\
\text { semana antes de los ensayos } \\
\text { experimentales. }\end{array}$ & $\begin{array}{l}\text { Participantes con trastornos } \\
\text { musculoesqueléticos. }\end{array}$ & $\begin{array}{c}\text { GRUPO CONTROL: } \\
\text { n: no refiere } \\
\text { GRUPO } \\
\begin{array}{c}\text { EXPERIMENTAL: } \\
\text { n: no refiere }\end{array}\end{array}$ & $\begin{array}{l}\text { Enfriamiento tópico } \\
\text { con compresas frías } \\
\text { (enfriamiento). }\end{array}$ & $\begin{array}{l}\text { Se realizaron cinco sesiones de aplicación } \\
\text { de compresas frías de } 15 \text { minutos al mús- } \\
\text { culo ejercitado a las } 0,3,24,48 \text { y } 72 \text { horas } \\
\text { después del dano muscular inducido por } \\
\text { el ejercicio excéntrico. }\end{array}$ & $\begin{array}{c}\text { Dolor, evaluado } \\
\text { con la Escala } \\
\text { Análoga Visual } \\
\text { (EAV). }\end{array}$ & $\begin{array}{l}\text { Daño muscu- } \\
\text { lar CK-MB y } \\
\text { mioglobina. }\end{array}$ & $\begin{array}{l}\text { Fuerza isométrica, } \\
\text { evaluado con Dinamó- } \\
\text { metro. }\end{array}$ & $\begin{array}{l}\text { El enfriamiento tópico es una in- } \\
\text { tervención clinica de uso comun, } \\
\text { no parece mejorar, sino que retrasa } \\
\text { la recuperación del daño muscular } \\
\text { inducido por el ejercicio excéntrico. }\end{array}$ \\
\hline $\begin{array}{c}\text { WILSON } \\
\text { 2017 } \\
\text { Reino Unido }\end{array}$ & 31 varones sanos & $\begin{array}{l}\text { Pertenecer a equipo de co- } \\
\text { rredores de resistencia en- } \\
\text { trenados. }\end{array}$ & $\begin{array}{l}\text { Ser fumadores y tener ante- } \\
\text { cedentes patológicos. }\end{array}$ & $\begin{array}{l}\text { GRUPO } \\
\text { EXPERIMENTAL: } \\
\text { n: no refiere }\end{array}$ & $\begin{array}{l}\text { Crioterapia de cuerpo } \\
\text { entero. }\end{array}$ & $\begin{array}{l}\text { Las percepciones de dolor muscular, es- } \\
\text { trés por entrenamiento y marcadores de la } \\
\text { función muscular se registraron antes de } \\
\text { la maratón y a las } 24 \text { y } 48 \mathrm{~h} \text { después del } \\
\text { ejercicio. Se tomaron muestras de sangre } \\
\text { al inció, después de la intervención } 242 \text { y } \\
48 \mathrm{~h} \text { después de la intervención para eva- } \\
\text { luarl la inflamación y el daño muscular }\end{array}$ & $\begin{array}{l}\text { Dolor, evalua- } \\
\text { do con Escala } \\
\text { Likert de 0 } \\
\text { (sin dolor en el } \\
\text { movimiento) a } \\
10 \text { (músculos } \\
\text { demasiado } \\
\text { doloridos para } \\
\text { moverse). }\end{array}$ & $\begin{array}{l}\text { Concentra- } \\
\text { ción de crea- } \\
\text { tina quinasa } \\
\text { CK. }\end{array}$ & $\begin{array}{l}\text { Torsión máxima y } \\
\text { contracciones iso- } \\
\text { métricas. Evaluadas } \\
\text { con Dinamómetro } \\
\text { isocinético. }\end{array}$ & 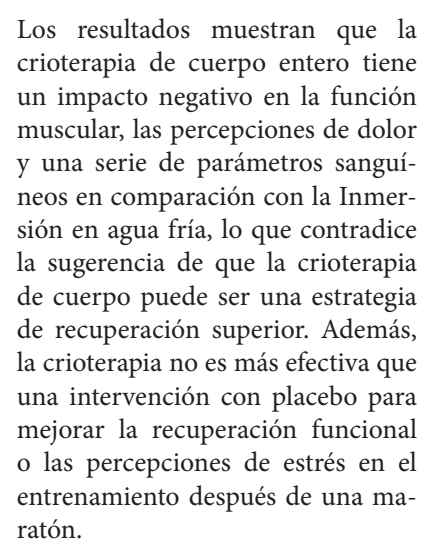 \\
\hline
\end{tabular}




\begin{tabular}{|c|c|c|c|c|c|c|c|c|c|c|}
\hline $\begin{array}{c}\text { AUTOR - AÑO } \\
\text {-PAIIS }\end{array}$ & $\begin{array}{c}\text { CARACTERÍSTICAS } \\
\text { DE LA POBLACIÓN } \\
\text { ESTUDIO }\end{array}$ & $\begin{array}{l}\text { CRITERIOS DE } \\
\text { INCLUSIÓN }\end{array}$ & $\begin{array}{c}\text { CRITERIOS DE } \\
\text { EXCLUSIÓN }\end{array}$ & INTE & ENCIÓN & PROTOCOLO/ TIEMPO DE MEDICIÓN & DOLOR & $\begin{array}{l}\text { MARCADORES } \\
\text { BIOQUÍMICOS }\end{array}$ & $\begin{array}{l}\text { RESULTADOS } \\
\text { SECUNDARIOS }\end{array}$ & $\begin{array}{c}\text { CONCLUSIÓN DEL } \\
\text { ESTUDIO }\end{array}$ \\
\hline $\begin{array}{l}\text { WILSON } \\
2018 \\
\text { Londres }\end{array}$ & $\begin{array}{l}24 \text { hombres entre- } \\
\text { nados }\end{array}$ & No reporta. & No reporta. & $\begin{array}{l}\text { GRUPO CONTROL: } \\
\text { n: } 15 \\
\end{array}$ & $\begin{array}{c}\text { Intervención placebo } \\
(\mathrm{PL})\end{array}$ & $\begin{array}{l}\text { Toma de muestras de sangre a los } 60 \text { y } 120 \\
\text { minutos después de la intervención. Los } \\
\text { particicantes rergresaron al laboratorio } \\
\text { para repetir mediciones de todos las va- } \\
\text { riables dependientes a las } 244,48 \text { y } 72 \text { h } \\
\text { tras la finalización de la sesion de entrena- } \\
\text { miento de resistencia. }\end{array}$ & DOMS & CK & No reporta. & 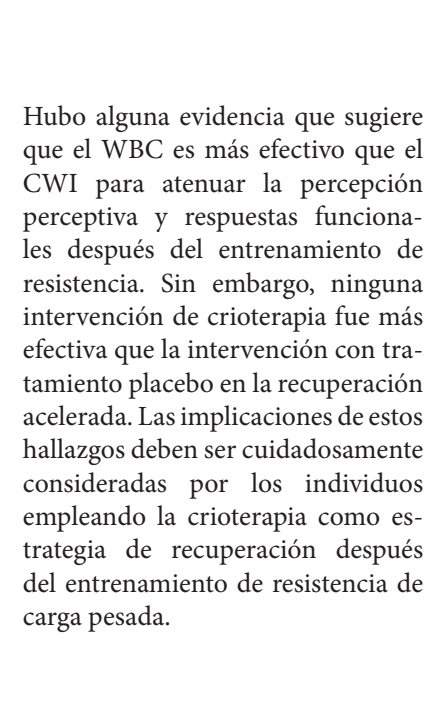 \\
\hline
\end{tabular}


sistemáticas, con el uso de la Review Manager versión 5.3. La evaluación del riesgo de sesgo de los ECA incluidos en la presente revisión sistemática (Figuras 2 y 3 ), se realizó también a través de una evaluación ciega con la participación nuevamente de la investigadora principal, la asesora y un fisioterapeuta externo del proyecto, este proceso también fue validado con el índice de Kappa.

Tabla 4. Evaluación de la calidad metodológica con la escala de PEDro $(n=10)^{*}$

\begin{tabular}{|c|c|c|c|c|c|c|c|c|c|c|c|}
\hline Referencia & P1 & P2 & P3 & P4 & P5 & P6 & P7 & P8 & P9 & P10 & Total \\
\hline Abaïdia 2017 (17) & + & - & + & - & - & - & + & + & + & + & $6 / 10$ \\
\hline Akley 2013 (18) & + & - & + & + & - & - & + & - & + & + & $6 / 10$ \\
\hline Andal 2013(19) & + & - & + & + & - & - & + & - & + & - & $5 / 10$ \\
\hline $\begin{array}{c}\text { Behringer } 2018 \\
(20)\end{array}$ & + & - & + & - & - & - & + & - & + & + & $5 / 10$ \\
\hline $\begin{array}{c}\text { De Marchi } 2017 \\
(21)\end{array}$ & + & - & + & - & - & - & + & - & + & + & $5 / 10$ \\
\hline De Paiva 2016 (22) & + & - & + & - & - & - & + & - & + & + & $5 / 10$ \\
\hline Fonseca 2016 (23) & + & - & + & - & - & - & + & - & + & + & $5 / 10$ \\
\hline Jakeman 2009 (24) & + & - & + & - & - & - & + & - & + & + & $5 / 10$ \\
\hline Missau 2018 (25) & + & - & + & - & - & - & + & - & + & + & $5 / 10$ \\
\hline Rowsell 2009 (26) & + & - & + & - & - & - & + & - & + & + & $5 / 10$ \\
\hline
\end{tabular}

PEDro (Physiotherapy Evidence Database): + Sí; - No. P1: Asignación aleatoria; P2: Ocultamiento de la asignación; P3: Grupos similares en línea de base; P4: Cegamiento de los participantes; P5: Cegamiento de los terapeutas; P6: Cegamiento el evaluador; P7: Abandonos < 15\%; P8: Análisis por intención a tratar; P9: Diferencias reportadas entre grupos; P10: Punto estimado y variabilidad reportada. * Evaluación reportada en PEDro (6).

Fuente: Las autoras 
Tabla 5. Evaluación de la calidad metodológica con la escala de PEDro $(n=9)$

\begin{tabular}{cccccccccccccccc}
\hline Referencia & P1 & P2 & P3 & P4 & P5 & P6 & P7 & P8 & P9 & P10 & Total & Kappa \\
\hline Ascensao 2011 (27) & + & - & + & - & - & - & - & - & + & + & & $4 / 10$ & 1 \\
\hline Bailey 2007 (28) & + & - & + & - & - & - & - & - & + & + & $4 / 10$ & 1 \\
\hline Crystal 2013 (29) & + & - & + & - & - & - & - & - & + & + & $4 / 10$ & 0.55 \\
\hline Goodall 2008 (30 & + & - & + & - & - & - & - & - & + & + & & \\
\hline
\end{tabular}

PEDro (Physiotherapy Evidence Database): + Sí; - No. P1: Asignación aleatoria; P2: Ocultamiento de la asignación; P3: Grupos similares en línea de base; P4: Cegamiento de los participantes; P5: Cegamiento de los terapeutas; P6: Cegamiento el evaluador; P7: Abandonos < 15\%; P8: Análisis por intención a tratar; P9: Diferencias reportadas entre grupos; P10: Punto estimado y variabilidad reportada. (6). Fuente: Las autoras

La evaluación de la calidad metodológica se realizó en 19 ECA de los cuales ningún estudio presenta clasificación $\geq 7$ en la escala propuesta por PEDro. Todos los estudios reportaron asignación aleatoria, grupos similares en línea base re- portaron las diferencias obtenidas entre grupos, así mismo, solo dos estudios tuvieron cegamiento de los participantes $(12,13)$. Un estudio realizó análisis por intención a tratar $(11,26)$, ningún artículo realizó cegamiento por el evaluador. 
Figura 2. Evaluación del riesgo de sesgo.

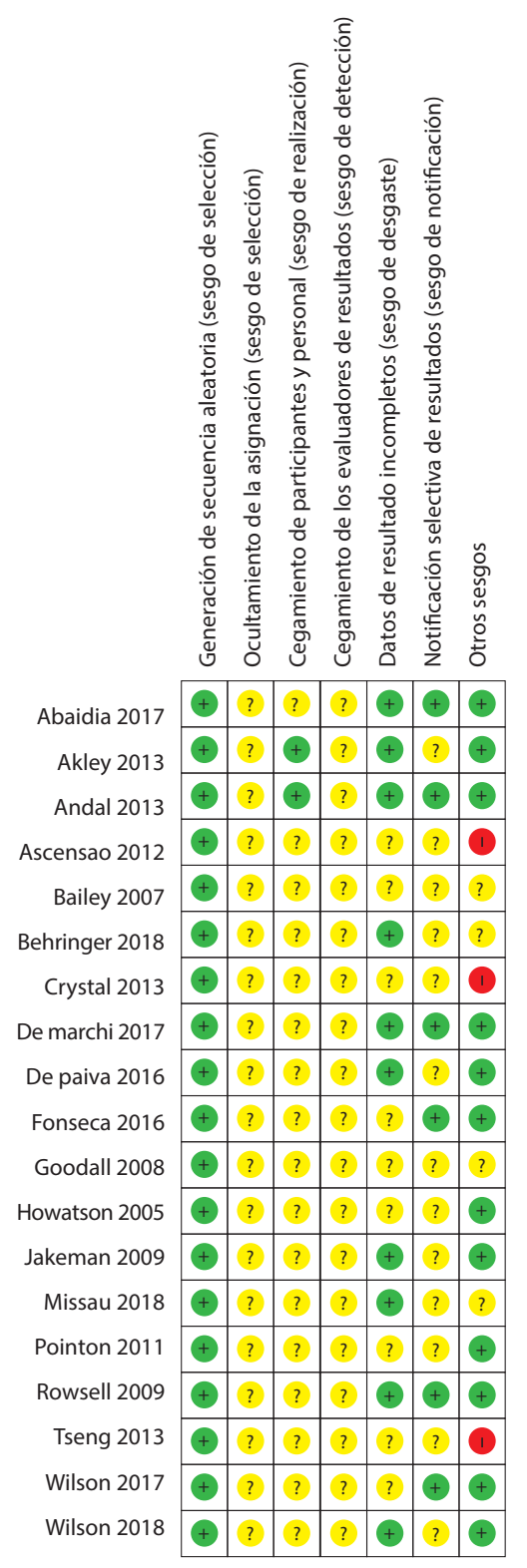

Figura 3. Resumen de la evaluación del riesgo de sesgo

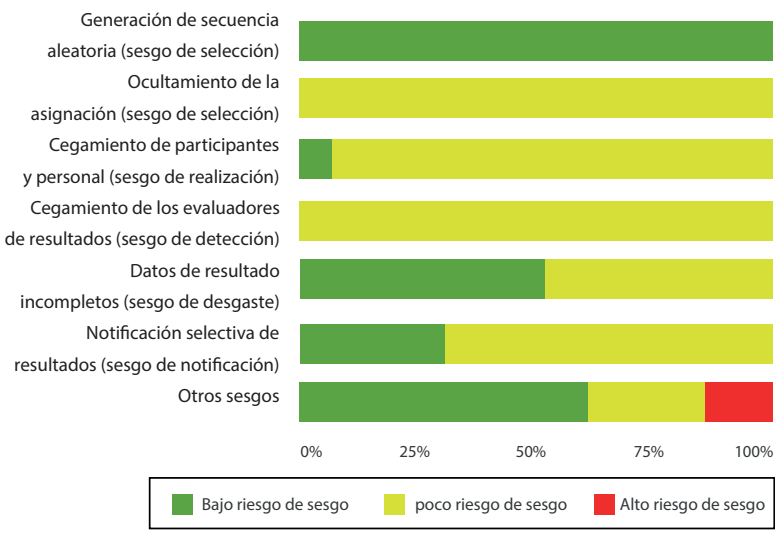

Respecto a la evaluación del riesgo de sesgo de los ECA incluidos, podemos concluir que en la totalidad de los estudios se reportó la generación de la secuencia aleatoria, sin embargo, no eran claros los métodos de asignación de la ocultación, así como en el blindaje de los resultados de la valoración, como se puede observar en las figuras 1 y 2 .

\section{Características de los Estudios Incluidos}

Se incluyen 19 artículos, publicados entre los años 2005 -2019, correspondientes a ECA en idioma inglés y portugués, la evaluación de la calidad metodológica según PEDro es en promedio de 5.6, los estudios fueron realizados en Francia - 
Abaidia (17), Australia - Pointon (5), Rowsell (26), Estados Unidos - Akley (18), Crystal (29), Brasil - Andal(19), De Marchi(21), Fonseca(23), Missau(25), Portugal - Ascensao(27), Reino Unido - Bailey(28), Goodall(30), Jakeman(24) Wilson(34), Alemania - Behringer(20), Londres De Paiva(22), Howatson(31), Wilson(35) y China - Tseng(33) (ver Tabla 5).

\section{Características de los Deportistas}

Se contó con un total de 205 personas activas, de ambos sexos, que realizaban algún tipo de práctica deportiva, predominando en los estudios de sexo masculino. La variable inicial que reportan los artículos son edad, peso y talla. La distribución de estas variables se encuentra con edades entre los 15 a 35 años, peso en kilogramos de $87.3 \pm$ 9.3 y talla en centímetros entre 1.74 a $1.82 \pm$ 3.6. Las prácticas deportivas incluían deportes como la lucha, fútbol, atletismo, basquetbol, taekwondo y béisbol. Dentro de los criterios de inclusión y exclusión que establecieron los estudios estaban: la firma del consentimiento informado y explicación de los riesgos, no presentar alteraciones del sistema musculo esquelético en etapa aguda y sistema cardiovascular, no presentar dolor, contraindicación médica de exposición al frío, no consumo de alcohol, cafeína, cigarrillo y medicamentos antiinflamatorios. Se les solicitó a los participantes que durante el estudio no practicaran actividad deportiva diferente al protocolo establecido para la investigación, así mismo, no realizar ejercicios polimétricos o ejercicios de resistencia 3 meses antes del estudio (ver Tabla 5).

\section{Características de las Intervenciones en Recuperación Muscular Post Ejercicio}

Los estudios contaban con grupo control y grupo experimental, sin embargo, dos estudios incluyeron más grupos para aplicar diferentes protocolos de intervención $(15,16)$; adicionalmente, algunos estudios no especificaron el tamaño de la muestra para los respectivos grupos. Respecto a la descripción de la fatiga muscular post esfuerzo, en hombres y mujeres activos que realizan práctica deportiva, se evidenció que la intervención basada en crioterapia fue aplicada con diferentes modalidades como: inmersión parcial o total en agua fría, crioterapia de cuerpo entero, masaje de hielo, crioterapia local con bolsas de hielo, fotobiomodulación (PBMT), compresas térmicas y crio cámara basada en nitrógeno gaseoso con una duración entre 3 a 30 minutos. De la misma manera, la temperatura varía según la modalidad encontrándose temperaturas de $-110^{\circ} \mathrm{C}$ hasta $-85^{\circ} \mathrm{C} 3$ a 4 minutos en crioterapia de cuerpo entero y $5^{\circ} \mathrm{C}$ hasta $10{ }^{\circ} \mathrm{C} 10$ a 20 minutos en inmersión total o parcial del cuerpo en agua fría, en donde a menor temperatura menor tiempo de exposición. Las intervenciones con las cuales compararon la crioterapia en sus diferentes modalidades fueron: recuperación pasiva, sin intervención, simulación 
de colocación de ultrasonido - placebo, reposo o descanso, drenaje linfático manual y ecografía simulada. El tiempo de medición fue, antes del protocolo e inmediatamente después, 24, 48, 72 $y$ a las 96 horas (ver Tabla 5).

\section{Medidas de Resultados Primarios}

Referente al resultado primario reportado por los ensayos clínicos se encontró que el elemento de la fatiga muscular (dolor muscular) fue evaluado a través de escalas Likert y la escala análoga visual, con diferentes rangos de entre 0 a 10 o 0 a 100, donde el valor mínimo corresponde a percepción baja y los valores máximos a percepción alta de dolor. Así mismo, fueron evaluados los marcadores bioquímicos a través de muestra sanguínea, a partir de los cuales reportaron: actividad de creatina quinasa CK, proteína de unión a ácidos grasos (h-FA BP), colesterol total, triglicéridos, neutrófilos, colesterol de lipoproteínas de alta densidad (HDLc), glucosa, ácido úrico, urea, fibrinógeno y aspartato aminotransferasa (AST). En la tabla 5. se muestran, de forma detallada, las conclusiones de los ECA participantes respecto a los cambios obtenidos post esfuerzo, luego de la aplicación de diferentes modalidades de la crioterapia.

\section{Medidas de Resultados Secundarios}

Referente a los resultados secundarios reportados por los ensayos clínicos se encontró que los ECA participantes determinan la evaluación de otros elementos en hombres y mujeres activas como: el salto vertical medido con diferentes protocolos como temporizador digital, tapete de fuerza, altura de salto; masa muscular en $\mathrm{Kg}$; inflamación determinada por cinta métrica antropométrica, rango de movimiento evaluado con goniometría, fuerza y contracción isométrica determinada a través de dinamometría, como variables que también se encuentran relacionadas con la fatiga muscular (Tabla 5).

\section{DISCUSIÓN}

Esta revisión sistemática describió intervenciones con crioterapia en fatiga muscular post esfuerzo específicamente en biomarcadores de daño muscular y el dolor en individuos que realizan práctica deportiva. Las modalidades encontradas fueron: inmersión total o parcial del cuerpo en agua fría, crioterapia de cuerpo entero, aplicación o masaje con hielo, paquetes fríos, bolsas de hielo, crio cámara basada en nitrógeno gaseoso, las cuales fueron comparadas entre sí y con otras técnicas utilizadas como la recuperación pasiva, placebo, drenaje linfático manual, fotobiomodulación, estiramientos estáticos y recuperación activa entre otros, modalidades que fueron aplicas posterior a la práctica deportiva. 
De acuerdo con los hallazgos, se estableció que la crioterapia en la modalidad de inmersión en agua fría, por si sola, genera mayores efectos en la disminución de la fatiga muscular, específicamente en el dolor post esfuerzo y disminución de la inflamación $(17,25)$; adicionalmente, genera cambios en los resultados de los marcadores bioquímicos a lo largo de 24,48 y 72 horas $(19,23)$, reduciendo el daño muscular (28) y posiblemente contribuyendo a una recuperación más rápida de la función neuromuscular (27); sin embargo, Goodall (30) mean y Jakeman (24) mencionan que después de realizar un ejercicio que generaba daño muscular, seguido de repetidas inmersiones en agua fría (CWI), no se observó ningún efecto positivo sobre biomarcadores como la creatinkinasa y dolor muscular.

De acuerdo con un estudio realizado por Wilson (34)this study aimed to assess the effects of whole body cryotherapy (WBC, la crioterapia de cuerpo entero(WBC) no mostró un impacto positivo en la disminución del dolor ni en los valores de biomarcadores sanguíneos en comparación con la inmersión en agua fría; en otras formas de aplicación como la crioterapia local o masaje con hielo, aunque el resultado fue positivo, el efecto en la disminución de la percepción del dolor fue mínimo y no se reportó evidencia de cambio específicamente en los marcadores de daño muscular (18,31-33). En este mismo sentido, Behringer (20) y Crystal (29) describieron, con respecto a los marcadores de daño muscular, que el uso del drenaje linfático manual, la crioterapia local o el descanso, aplicados por 30 minutos, no evidencian cambios significativos; así mismo, no encontraron efectos beneficiosos sobre la inflamación o el dolor muscular cuando se usa drenaje linfático manual y la crioterapia local en comparación con la recuperación pasiva.

En la revisión, De Marchi (21) y De Paiva (22) encontraron que la aplicación de crioterapia asociada a fotobioestimulación (PBMT) no mejora los efectos de la aplicación PBMT, siendo la aplicación aislada de PBMT una mejor opción para la recuperación muscular, tanto a corto como a largo plazo; por el contrario, hallaron que el uso de la crioterapia en aislamiento fue incapaz de proporcionar recuperación muscular, por tanto, establecen la necesidad de estudios de campo adicionales que determinen los parámetros de dosis según nivel de práctica deportiva: deportistas élite, recreativos entre otros.

La intervención basada en Crioterapia se realizó con diferentes modalidades como inmersión parcial o total en agua fría, crioterapia de cuerpo entero, masaje de hielo, crioterapia local con bolsas de hielo, fotobiomodulación (PBMT), compresas térmicas y crio cámara basada en nitrógeno gaseoso; en estos casos se varió la temperatura según la modalidad, con lo cual se encontraron temperaturas de $-10^{\circ} \mathrm{C},-60^{\circ} \mathrm{Cy}-110^{\circ} \mathrm{C}$ por $3 \mathrm{~min}$ 
en (WBC) y de $5^{\circ} \mathrm{C}$ a $15^{\circ} \mathrm{C}$ por 10 a 20 min en $\mathrm{WCl}$. (a menor temperatura menor tiempo de exposición). Las intervenciones con las cuales compararon la crioterapia fueron: recuperación pasiva, sin intervención, placebo, drenaje linfático manual y fotobiomodulación PBMT. Los tiempos de medición fueron: antes del protocolo e inmediatamente después, 1, 24, 48, 72 y a las 96 horas.

Con respecto a los protocolos de ejercicio para inducir fatiga y daño muscular, se encontraron estudios que no reportaron el tipo de ejercicio realizado $(6,17,21,23,24,29)$; otros protocolos consistieron en realizar contracciones excéntricas y concéntricas de diferentes grupos musculares (flexores de codo, flexores y extensores de rodilla) $(20,22,25,30-33)$ adicionalmente, se reportaron protocolos con ejercicio de resistencia como la maratón $(34,35)$, intervenciones realizadas posteriores a un partido de fútbol (27), para 4 partidos jugados en 4 días con 24 horas entre ellos (26) e intervenciones posteriores a un combate $(18,19)$.

\section{Limitaciones}

Los autores expresan como limitación el bajo promedio obtenido en el puntaje de la evaluación de la calidad metodológica, según PEDro (5.6 sobre el valor total propuesto por PEDro de 10), en diez (10) de los ECAS incluidos.
Adicionalmente, se encontró heterogeneidad clínica para los procesos de evaluación e intervención tales como participación en ECAS según sexo, modo o tipo de práctica deportiva, nivel de entrenamiento y protocolos de ejercicio para inducir fatiga o daño muscular; lo anterior, se constituye en una limitación debido a que la eficiencia de los diferentes medios o modalidades de recuperación estaría estrechamente ligada con el tipo de fatiga, el ejercicio previo o las cargas de entrenamiento, entre otras, generándose una dificultad para los autores en el establecimiento definitivo de la conclusión, la cual consideramos fundamental a la hora de tomar una decisión de aplicación clínica frente al uso de la crioterapia, como medio para disminuir la fatiga y el dolor muscular.

\section{Aplicaciones Clínicas}

La práctica del fisioterapeuta, y en general de las ciencias aplicadas al deporte en el quehacer cotidiano, exige la toma de decisiones permanentes en pro de lo que su objeto de estudio le demanda, por esto, la evidencia científica se debe constituir como una herramienta fundamental para que esta toma de decisiones sea la acertada, de acuerdo con la condición del usuario, su pronóstico, diagnóstico y la intervención que se le brinde. La evidencia científica encontrada expone la necesidad de realizar ensayos clínicos aleatorizados con mayor rigor metodológico, con 
el objeto de poder evidenciar los cambios producidos por la aplicación de la crioterapia en sus diferentes modalidades $y$, de esta forma, sustentar científicamente el quehacer de los profesionales.

\section{CONCLUSIÓN}

La crioterapia, en la modalidad de inmersión total o parcial del cuerpo en agua fría, por sí sola genera mejores efectos en la disminución de la fatiga muscular, específicamente en el dolor post esfuerzo y cambios en los resultados sanguíneos de los marcadores bioquímicos. Es importante que esta inmersión se haga inmediatamente después del esfuerzo o en las 24 horas siguientes, a temperaturas que oscilen entre $5^{\circ} \mathrm{C}$ y $10^{\circ} \mathrm{C}$ durante 10 a 20 minutos; resaltando que a menor temperatura menor tiempo de exposición.

Existen otras modalidades de aplicación como la crioterapia de cuerpo entero, la crioterapia local, el masaje con hielo, el drenaje linfático manual, masaje sedativo y la recuperación pasiva, entre otras, las cuales reportan mejoras en la disminución del dolor y la percepción de la fatiga muscular, pero no en la respuesta de los biomarcadores de daño muscular. Estas modalidades o medios terapéuticos muestran resultados positivos en la disminución del dolor y percepción de la fatiga muscular, siempre y cuando su aplicación sea combinada para realizar un abordaje integral. Respecto al manejo individual de las modalidades anterior- mente mencionadas, cabe anotar que sirven para tratar sintomatología específica de diferentes patologías y/o lesiones deportivas.

\section{CONFICTO DE INTERESES}

Las autoras declaran que no existe conflicto de intereses.

\section{FINANCIACIÓN}

La investigación se financió con recursos propios de los investigadores.

\section{REFERENCIAS}

1. Cañizares M, JM. La actividad física y el deporte en la sociedad actual . 2017. Editorial W. 2017. 2017 p.32

2. Cárdenas D, Conde-González J, Perales JC. La fatiga como estado motivacional subjetivo. Revista Andaluza de Med del Deport . 2019;43(2S):176.

3. Balius R, Pedret C. Lesiones Musculares en el Deporte. Editorial Médica Panamericana. 2013.

4. Dupuy O, Douzi W, Theurot D, Bosquet L, Dugué B. An evidence-based approach for choosing post-exercise recovery techniques to reduce markers of muscle damage, Soreness, 
fatigue, and inflammation: A systematic review with meta-analysis. Front Physiol. 2018;9:1-15. https://doi.org/10.3389/fphys.2018.00403

5. Hohenauer E, Taeymans J, Baeyens JP, Clarys P, Clijsen R. The effect of post-exercise cryotherapy on recovery characteristics: A systematic review and meta-analysis. PLoS One. 2015;10(9):1-23. https://doi.org/10.1371/journal.pone.0139028

6. Torres R, Ribeiro F, Alberto Duarte J, Cabri JMH. Evidence of the physiotherapeutic interventions used currently after exercise-induced muscle damage: Systematic review and meta-analysis. Phys Ther Sport. 2012;13(2):101-14. https:// doi.org/10.1016/j.ptsp.2011.07.005

7. González Ravé JM, Pablos Abella C, Navarro Valdivielso F. Entrenamiento Deportivo. Teoría y práctica. Editorial Médica Panamericana. 2014. 4-8 p.

8. Sandoval MC, Herrera E, Camargo DM. Efecto de tres modalidades de crioterapia sobre la temperatura de la piel durante las fases de enfriamiento y recalentamiento Effects of three cold modalities on skin temperature during the cooling and rewarming phases. Rev. Univ. Ind. Santander. Salud. 2011;43(2):119-29.

9. Gutiérrez-Espinoza HJ, Lavado- Bustamante IP, Méndez-Pérez SJ. Systematic review of the analgesic effect of cryotherapy in the manage- ment of musculoskeletal pain. Rev la Soc Esp del Dolor. 2010;17(5):242-52.

10. Robinson KA, Dickersin K. Development of a highly sensitive search strategy for the retrieval of reports of controlled trials using PubMed. Int J Epidemiol. 2002;31(1):150-3. https://doi. org/10.1093/ije/31.1.150

11. Urrútia G, Bonfill X. Declaración PRISMA: una propuesta para mejorar la publicación de revisiones sistemáticas y metaanálisis. Medicina Clínica. 2010;135:507-11. https://doi.org/10.1016/j.medcli.2010.01.015

12. Centre of Evidence-Based Physiotherapy C. Escala PEDro. Georg Inst Glob Heal. 2012;1-2.

13. Liberati A, Altman DG, Tetzlaff J, Mulrow C, Gøtzsche PC, loannidis JPA, et al. The PRISMA statement for reporting systematic reviews and meta-analyses of studies that evaluate health care interventions: explanation and elaboration. J Clin Epidemiol. 2009;62(10):e1-34. https://doi.org/10.1371/journal.pmed.1000100

14. Centro Cochrane Iberoamericano, traductores. Manual Cochrane de Revisiones Sistemáticas de Intervenciones, versión 5.1.0. 2012;(March):1-639.

15. Landis JR, Koch GG. The Measurement of Observer Agreement for Categorical Data. Biometrics. 1977;33(1):159-74. 
16. Herbert R, Moseley A, Sherrington C, Maher C. Escala de Pedro. Evidencia científica. Physiotherapy. 2000;86(1):55.

17. Abaïdia A-E, Delecroix B, Baquet G, Dupont G, Lamblin J, Leduc $C$, et al. Recovery from exercise-induced muscle damage: Cold-water immersion versus whole-body cryotherapy. Int J Sports Physiol Perform. 2017;12(3):402-9. https://doi.org/10.1123/ijspp.2016-0186

18. Oakley ET, Pardeiro RB, Powell JW, Millar AL. The effects of multiple daily applications of ice to the hamstrings on biochemical measures, signs, and symptoms associated with exerciseinduced muscle damage. J Strength Cond Res. 2013;27(10):2743-51. https://doi.org/10.1519/ JSC.0b013e31828830df

19. Pinho EA, Brito CJ, Costa WO, Nardelli C, Lacerda E, Franchini E. Influence of cryotherapy on muscle damage markers in jiu-jitsu fighters after competition: a cross-over study. Rev Andal Med Deporte. 2014;7(1):7-12

20. Behringer $M$, Jedlicka $D$, Mester J. Effects of lymphatic drainage and cryotherapy on indirect markers of muscle damage. J Sports Med Phys Fitness. 2018;58(6):903-9. https://doi. org/10.23736/S0022-4707.17.07261-9

21. De Marchi T, Schmitt VM, Machado GP, de Sene JS, de Col CD, Tairova O, et al. Does pho- tobiomodulation therapy is better than $\mathrm{cr}$ yotherapy in muscle recovery after a high-intensity exercise? A randomized, double-blind, placebo-controlled clinical trial. Lasers Med Sci. 2017;32(2):429-37. https://doi.org/10.1007/ s10103-016-2139-9

22. de Paiva PRV, Tomazoni SS, Johnson DS, Vanin AA, Albuquerque-Pontes GM, Machado C dos SM, et al. Photobiomodulation therapy (PBMT) and/or cryotherapy in skeletal muscle restitution, what is better? A randomized, double-blinded, placebo-controlled clinical trial. Lasers Med Sci. 2016;31(9):1925-33. https:// doi.org/10.1007/s10103-016-2071-z

23. Fonseca LB, Brito CJ, Silva RJS, Silva-Grigoletto $M E$, da Silva WM, Franchini $E$. Use of Cold-Water Immersion to Reduce Muscle Damage and Delayed-Onset Muscle Soreness and Preserve Muscle Power in Jiu-Jitsu Athletes. J Athl Train. 2016;51(7):540-9. https://doi. org/10.4085/1062-6050-51.9.01

24. Jakeman JR, Macrae R, Eston R. A single 10-min bout of cold-water immersion therapy after strenuous plyometric exercise has no beneficial effect on recovery from the symptoms of exercise-induced muscle damage. Ergonomics. 2009;52(4):456-60. https://doi. org/10.1080/00140130802707733 
25. Missau E, da Silva AMV, Signori LU, Teixeira A de O, Franco OS, Martins CN, et al. Cold water immersion and inflammatory response after resistance exercises. Rev Bras Med do Esporte. 2018;24(5):372-6. https://doi. org/10.1590/1517-869220182405182913

26. Rowsell GJ, Coutts AJ, Reaburn P, Hill-Haas S. Effects of cold-water immersion on physical performance between successive matches in high-performance junior male soccer players. J Sports Sci. 2009;27(6):565-73. https://doi. org/10.1080/02640410802603855

27. Ascensão A, Leite $M$, Rebelo AN, Magalhäes $S$, Magalhäes J. Effects of cold water immersion on the recovery of physical performance and muscle damage following a one-off soccer match. J Sports Sci. 2011;29(3):217-25. https:// doi.org/10.1080/02640414.2010.526132

28. Bailey DM, Erith SJ, Griffin PJ, Dowson A, Brewer DS, Gant N, et al. Influence of cold-water immersion on indices of muscle damage following prolonged intermittent shuttle running. J Sports Sci. 2007;25(11):1163-70. https://doi. org/10.1080/02640410600982659

29. Crystal NJ, Townson DH, Cook SB, Laroche DP. Effect of cryotherapy on muscle recovery and inflammation following a bout of damaging exer- cise. Eur J Appl Physiol. 2013;113(10):2577-86. https://doi.org/10.1007/s00421-013-2693-9

30. Goodall S, Howatson G. The effects of multiple cold water immersions on indices of muscle damage. J Sport Sci Med. 2008;7(2):235-41.

31. Howatson G, Gaze D, Van Someren KA. The efficacy of ice massage in the treatment of exercise-induced muscle damage. Scand J Med Sci Sport. 2005;15(6):416-22. https://doi.org/10.1111/j.1600-0838.2005.00437.x

32. Pointon M, Duffield R, Cannon J, Marino FE. Cold application for neuromuscular recovery following intense lower-body exercise. Eur J Appl Physiol. 2011;111(12):2977-86. https:// doi.org/10.1007/s00421-011-1924-1

33. Tseng CY, Lee JP, Tsai SD, Kao CL, Liu TC, et al. Topical cooling (icing) delays recovery from eccentric exercise-induced muscle damage. J Strength Cond Res. 2013;27(5):1354-61. https:// doi.org/10.1519/JSC.0b013e318267a22c

34. Wilson D, Cockburn E, Paice K, Sinclair S, Faki T, Hills FA, et al. Recovery following a marathon: a comparison of cold water immersion, whole body cryotherapy and a placebo control. Eur J Appl Physiol. 2017;118(1):153-63. 
Lyda F Vallejo-Castillo, Carolina Sandoval-Cuellar, Aura C Quino- Ávila

35. Wilson $\sqcup$, Dimitriou L, Hills FA, Gondek MB, Cockburn E. Whole body cryotherapy, cold water immersion, or a placebo following resistance exercise: a case of mind over matter? Eur J Appl Physiol. 2019;119(1):135-47. https:// doi.org/10.1007/s00421-017-3757-z

\section{(ब) $(\AA ⿻$}

Esta obra está bajo una licencia de Creative Commons Reconocimiento-NoComercial 4.0 Internacional 